\title{
An operator perturbation method for polarized line transfer
}

\section{Generalized PALI method for Hanle effect with partial frequency redistribution and collisions}

\author{
D. M. Fluri ${ }^{1}$, K. N. Nagendra ${ }^{2,3}$, and H. Frisch ${ }^{3}$ \\ ${ }^{1}$ Institute of Astronomy, ETH Zentrum, 8092 Zurich, Switzerland \\ 2 Indian Institute of Astrophysics, Bangalore 560 034, India \\ ${ }^{3}$ Laboratoire G. D. Cassini (CNRS, UMR 6529), Observatoire de la Côte d'Azur, BP 4229, 06304 Nice Cedex 4, France
}

Received 28 August 2001 / Accepted 5 December 2002

\begin{abstract}
A generalized iteration method is presented to solve the polarized line transfer equation for a two-level-atom in an arbitrarily oriented, weak magnetic field. The polarized redistribution matrix employed accounts self-consistently for collisions as well as the presence of a weak magnetic field responsible for the Hanle effect. The proposed numerical method of solution is based on a Polarized Approximate Lambda Iteration (PALI) method. A Fourier decomposition of the radiation field and of the phase matrix with respect to the azimuthal angle reduces the complexity of the problem. A generalized core-wing technique is proposed, which permits an efficient implementation of the frequency domain structure inherent in the polarized redistribution matrix. The numerical method is tested for its accuracy and efficiency by comparing with the existing methods.
\end{abstract}

Key words. line: formation - polarization - radiative transfer - scattering - methods: numerical - Sun: atmosphere

\section{Introduction}

Line polarization produced by scattering processes has developed into a topic of great interest in solar physics (see Stenflo \& Nagendra 1996; Nagendra \& Stenflo 1999). Systematic observations by Stenflo et al. (1983a,b) and theoretical attempts to model them proved that the scattering polarization and quantum interference effects are the basic causes of line polarization in the spectra from quiet regions. The structural richness of the so called second solar spectrum is revealed in observations by Stenflo \& Keller $(1996,1997)$ and in the atlas recently published by Gandorfer (2000), which for the first time presents an overview of the scattering polarization in the whole visible wavelength range with a sensitivity well below $10^{-4}$. Resonance scattering between bound atomic states is responsible for the linear polarization in spectral lines. The modification of this polarization by the presence of a weak magnetic field is called the Hanle effect. The Hanle effect in scattering polarization is an important tool to diagnose the smallscale structure of spatially unresolved magnetic fields (Stenflo 2001; Stenflo et al. 2001). For a review of the Hanle effect see Faurobert-Scholl (1996), Frisch (1999), and Trujillo Bueno (2001). In recent years the Hanle effect has also become a diagnostic tool in stellar astrophysics (see Ignace et al. 1997, 1999; Ignace 2001). An interpretation of the high spectral resolution data on the Hanle effect requires the solution of the Hanle

Send offprint requests to: D. M. Fluri,

e-mail: dfluri@astro.phys.ethz.ch effect line transfer problem with a correct treatment of partial frequency redistribution (PRD). A conventional direct solution of this problem requires large computational resources. Hence there is a need to develop efficient iterative methods to solve this problem.

The challenges in modelling scattering polarization constitutes not only the development of faster numerical methods, but also obtaining theoretical expressions for the redistribution matrix that describes the correlation between frequency, direction of propagation, and state of polarization of incident and scattered photons. Recently Bommier (1997a,b) has derived selfconsistent polarized redistribution matrices for the Hanle effect. The purpose of this paper is to adopt these matrices into a generalized PALI method for Hanle PRD problems.

Scalar (unpolarized) frequency redistribution functions for two-level atoms, neglecting collisions, were first studied by Hummer (1962). More general expressions for collisional frequency redistribution on resonance and subordinate lines were derived by Oxenius (1965), Heinzel (1981), Hubeny (1982, 1985, and references therein), Hubeny et al. (1983a,b), Hubeny \& Cooper (1986), and Hubeny \& Lites (1995).

The problem of polarized scattering in non-magnetic media with PRD was first addressed by Omont et al. (1972). Rees \& Saliba (1982) proposed a decoupling of frequency redistribution from the phase matrix for resonance scattering - an approximation which has turned out to be practical and useful. Many authors have subsequently employed this approximation (see, Faurobert 1987; Nagendra 1988; Frisch 1999, and 
references therein). The phase matrix describes the correlations between direction of propagation and state of polarization of absorbed and re-emitted photons. A general expression for the redistribution matrix accounting for collisions in the absence of a magnetic field was derived by Domke \& Hubeny (1988) and Streater et al. (1988) and used in non-magnetic line transfer computations by Nagendra (1994, 1995). The effects of weak magnetic fields on the polarized redistribution matrix were first considered by Omont et al. (1973). The polarized line transfer problem for the Hanle effect with conventional treatment of PRD was first solved by Faurobert-Scholl (1991). In an extension of this method she used a heuristic approach based on the redistribution matrix of Domke \& Hubeny (1988) to account for collisions in weak magnetic fields. Studies of the solar Ca I $4227 \AA$ and Sr I $4607 \AA$ lines were undertaken by Faurobert-Scholl (1992, 1993), and Faurobert-Scholl et al. (1995) using this approach, in order to explain the linear polarization in these lines.

The formulation of polarized line transfer developed in the last two decades has followed two different approaches to the Hanle effect problem. The first one (also pursued in the present paper) is a scattering formalism that makes use of vector differential and integral equations for polarized line transfer. The second approach employs the theoretical framework developed by Landi Degl'Innocenti (1983, 1984, 1985), which is based on the irreducible tensor components of the atomic density matrix (see also Bommier \& Sahal-Bréchot 1978). This formalism lends itself easily to multi-level atoms including lower-level atomic polarization. Frisch $(1998,1999)$ has shown that the two approaches are equivalent in the case of a two-level atom with complete frequency redistribution (CRD).

The scattering formalism has been implemented into a PALI method (Faurobert-Scholl et al. 1997; Paletou \& Faurobert-Scholl 1997; Nagendra et al. 1998, 1999, 2000, henceforth referred to as Paper I, II, III, IV, and V, respectively). The iteration scheme is similar to the scalar Approximate Lambda Iteration (ALI) methods using a local approximate operator (Olson et al. 1986). PALI makes use of the reduced transfer equation derived through an azimuthal Fourier decomposition of the radiation field as well as of the Hanle phase matrix. This greatly decreases the CPU time and memory requirements compared to direct methods like the Feautrier method (Faurobert-Scholl 1991) or the Discrete Space Method (Nagendra 1988). Paper I deals with CRD and Paper II with $\mathrm{PRD}$ in resonance line scattering in non-magnetic media, whereas Paper III (for CRD) and Papers IV and V (for PRD) include the presence of weak magnetic fields. Note that in the absence of a complete theoretical framework, a phenomenological expression for the Hanle redistribution matrix based on heuristic arguments was implemented in all these papers. The scattering formalism has also been used by Dittman (1999) for 3D polarized radiative transfer (not based on PALI).

The density matrix formalism has been applied to develop very efficient operator splitting methods for Non-LTE polarized transfer problems in multidimensional media and for multi-level atoms (Trujillo Bueno \& Manso Sainz 1999; Manso Sainz \& Trujillo Bueno 1999; Fabiani Bendicho \& Trujillo Bueno 1999). The formalism can naturally consider lower-level atomic polarization (Trujillo Bueno \& Landi Degl'Innocenti 1997) as has been successfully demonstrated through the computations of the Ca II infrared triplet and the multiplet no. 2 of $\mathrm{Mg}_{\mathrm{I}}$ (Manso Sainz \& Trujillo Bueno 2001; Trujillo Bueno 2001) to model observations by Stenflo et al. (2000). The standard theory of the density matrix formalism works for the approximation of CRD. Landi Degl'Innocenti et al. (1997) have developed a theory of Rayleigh scattering in the presence of magnetic fields of arbitrary strength but for a no-collision case based on metalevels for describing coherent scattering. With this theory Landi Degl'Innocenti (1998) could partly explain the linear polarization features of the $\mathrm{Na}_{\mathrm{I}} \mathrm{D}_{2}$ and $\mathrm{D}_{1}$ lines.

Bommier (1997a,b) has derived a general theory for Rayleigh scattering in the presence of magnetic fields of arbitrary strength. Her treatment, based on quantum-field theory in the weak radiation field limit, takes into account the effects of elastic and inelastic collisions self-consistently in a two-level picture. The corresponding classical oscillator theory proposed by Bommier \& Stenflo (1999) gives identical results as the quantum theory for normal Zeeman triplets and has the advantage of being conceptually more transparent.

In the present paper we introduce a fast numerical method that implements the self-consistent redistribution theory of Bommier (1997a,b). We assume that the magnetic fields are weak (the Hanle effect regime) and further work with angle averaged frequency redistribution functions, which corresponds to the approximation level III of Bommier (1997b). This approximation has been implemented by Faurobert-Scholl et al. (1999) for pure $R_{\mathrm{II}}$ frequency redistribution using a perturbation method. The method presented in this paper is based on the PALI code of Nagendra et al. (1999) and has the advantage of being very fast and highly economic regarding memory requirement.

In Sect. 2 we formulate the radiative transfer problem followed in Sect. 3 by an extensive description of the numerical method of solution. In Sect. 4, which is devoted to the testing of the method, we discuss the convergence properties of the code, check how well flux conservation is satisfied in conservative scattering, and compare the results with independent methods.

\section{Formulation of the radiative transfer problem}

\subsection{Assumptions}

We have solved the polarized line transfer equation with partial frequency redistribution in weak magnetic fields. The following assumptions are made:

- 2-level-atom model;

- unpolarized lower level;

- approximation level III of Bommier (1997b) involving angle averaged $R_{\mathrm{II}}$ and $R_{\mathrm{III}}$ frequency redistribution functions, and the frequency domain structures;

- weak radiation field limit (i.e. stimulated emission is neglected with respect to spontaneous emission);

- unpolarized background continuum;

- no continuum scattering;

- no circular polarization. 


\subsection{Polarized line transfer equation for the Hanle effect}

The one-dimensional line transfer equation for the Stokes vector $\mathscr{I}=(I, Q, U)^{\mathrm{T}}$ may be written as

$$
\begin{aligned}
& \mu \frac{\partial \mathscr{I}(\tau, x, \boldsymbol{n})}{\partial \tau}= \\
& \quad(\phi(\tau, x)+\beta(\tau))[\mathscr{I}(\tau, x, \boldsymbol{n})-\mathscr{S}(\tau, x, \boldsymbol{n})],
\end{aligned}
$$

where $\tau$ is the monochromatic optical depth, $x$ is the frequency in units of a standard Doppler width $(x=0$ at line center), $\phi$ is the profile function, $\beta$ is the ratio of continuum to integrated line absorption coefficient, and $\boldsymbol{n}$ the propagation direction. Stokes $V$, representing circular polarization, is not regarded since for the Hanle effect the transfer equation for $V$ is fully decoupled from the transfer equation given above for the other three Stokes parameters. The total source vector $\mathscr{S}=\left(S_{I}, S_{Q}, S_{U}\right)^{\mathrm{T}}$ is given by

$$
\mathscr{S}(\tau, x, \boldsymbol{n})=\frac{\phi(\tau, x) \mathscr{S}_{\ell}(\tau, x, \boldsymbol{n})+\beta(\tau) \mathscr{B}_{\mathrm{th}}(\tau)}{\phi(\tau, x)+\beta(\tau)},
$$

where $\mathscr{B}_{\text {th }}(\tau)=\left(B_{v_{0}}, 0,0\right)^{\mathrm{T}}$ with $B_{v_{0}}$ the Planck function. The line source vector $\mathscr{S}_{\ell}$ has the form

$$
\begin{aligned}
& \mathscr{S}_{\ell}(\tau, x, \boldsymbol{n})=\epsilon \mathscr{B}_{\mathrm{th}}(\tau) \\
& +\frac{1}{\phi(\tau, x)} \int_{-\infty}^{+\infty} \mathrm{d} x^{\prime} \oint \frac{\mathrm{d} \Omega^{\prime}}{4 \pi} \hat{\mathscr{R}}\left(x, x^{\prime}, \boldsymbol{n}, \boldsymbol{n}^{\prime}, \boldsymbol{B}\right) \mathscr{I}\left(\tau, x^{\prime}, \boldsymbol{n}^{\prime}\right),
\end{aligned}
$$

where $\epsilon$ is the thermalization parameter. The quantity $\hat{\mathscr{R}}$ gives the redistribution matrix, and $\boldsymbol{B}$ represents the magnetic field vector. To distinguish matrices from column vectors, matrices are marked with a hat over the concerned quantity in the whole paper.

\subsection{Redistribution matrix and the domain structure}

In this section we define explicitly the redistribution matrix and explain the meaning of frequency domains. We use the redistribution matrix derived by Bommier (1997b), particularly the one listed under approximation level III (see Eqs. (103) to (113) in Bommier 1997b). It is necessary that we rewrite the full expressions of the redistribution matrix of Bommier (1997b) to introduce the notation and because we have to rearrange the various terms to suit the numerical method presented in Sect. 3.

Approximation level III of Bommier (1997b) refers to the use of angle averaged scalar redistribution functions. The 2D frequency space $\left(x, x^{\prime}\right)$, where $x$ and $x^{\prime}$ denote outgoing and incoming frequencies respectively, is divided into different regions called frequency domains. Within each domain in $\left(x, x^{\prime}\right)$-space the redistribution matrix can be written as a linear combination of terms which are products of scalar redistribution functions and phase matrices. The scalar redistribution functions are $R_{\mathrm{III}}\left(x, x^{\prime}\right)$ and $R_{\mathrm{II}}\left(x, x^{\prime}\right)$ in the standard notation (Hummer 1962) and the phase matrices represent Hanle and Rayleigh scattering.
The total redistribution matrix can be written as a sum of two parts

$$
\begin{aligned}
\hat{\mathscr{R}}\left(x, x^{\prime}, \boldsymbol{n}, \boldsymbol{n}^{\prime}, \boldsymbol{B}\right)= & \hat{\mathscr{R}}_{\mathrm{III}}\left(x, x^{\prime}, \boldsymbol{n}, \boldsymbol{n}^{\prime}, \boldsymbol{B}\right) \\
& +\hat{\mathscr{R}}_{\mathrm{II}}\left(x, x^{\prime}, \boldsymbol{n}, \boldsymbol{n}^{\prime}, \boldsymbol{B}\right) .
\end{aligned}
$$

The first part of the sum consists of linear combinations of the terms written as products of $R_{\mathrm{III}}$ with the phase matrices, while the second part consists of similar products involving $R_{\mathrm{II}}$. We look at the two parts separately as they have different frequency domain structures associated with them.

\section{Physical frequency domains}

For easier reference we introduce a numbering of the frequency domains, which is shown graphically in Fig. 1. We have a total of five frequency domains, where domain no. 1 to 3 are associated with $\hat{\mathscr{R}}_{\text {III }}$, and domain no. 4 and 5 are associated with $\hat{\mathscr{R}}_{\mathrm{II}}$. We will refer to these frequency domains as "physical domains", where the word "physical" stresses the fact that the domain structure has been obtained directly from the formulation of Bommier (1997b). In contrast, we will introduce "approximated domains" in Sect. 3.2.1, which are used in one single step of the numerical method and represent a numerical simplification of the physical domains.

The structure of the physical frequency domains for $\hat{\mathscr{R}}_{\mathrm{III}}$ (see panel (a) of Fig. 1), is obtained according to the following logical sequence (Bommier 1997b):

$$
\text { If } \begin{aligned}
& \left\{z v_{\mathrm{c}}(a)\left|x^{\prime}\right|-\left(x^{2}+x^{\prime 2}\right)<(z-1) v_{\mathrm{c}}^{2}(a)\right. \\
& \text { and } z v_{\mathrm{c}}(a)|x|-\left(x^{2}+x^{\prime 2}\right)<(z-1) v_{\mathrm{c}}^{2}(a) \\
& \text { and }\left|x^{\prime}\right|<\sqrt{2} v_{\mathrm{c}}(a) \\
& \text { and } \left.|x|<\sqrt{2} v_{\mathrm{c}}(a)\right\}
\end{aligned}
$$

\section{then: domain 1}

$$
\text { else, if } \quad\left\{|x|<v_{\mathrm{c}}(a) \text { or }\left|x^{\prime}\right|<v_{\mathrm{c}}(a)\right\}
$$

\section{then: domain 2}

\section{else: domain 3}

endif.

In the above equations we have used the quantity $z=2 \sqrt{2}+2$. The variable $a$ is the damping parameter. The cut-off parameter $v_{\mathrm{c}}(a)$ is defined as in Bommier (1997a) and represents the frequency at the transition of the Voigt profile from the Gaussian core to the Lorentzian wing (cf. Eq. (85) and Table 2 in Bommier 1997b).

For $\hat{\mathscr{R}}_{\text {II }}$ the two-dimensional $\left(x, x^{\prime}\right)$-space divides into two physical domains, shown in panel (b) of Fig. 1, according to the following logic (Bommier 1997b):

If $\quad\left\{x\left(x+x^{\prime}\right)<2 v_{\mathrm{c}}^{2}(a)\right.$ and $\left.x^{\prime}\left(x+x^{\prime}\right)<2 v_{\mathrm{c}}^{2}(a)\right\}$

then: domain 4

else: domain 5

endif. 

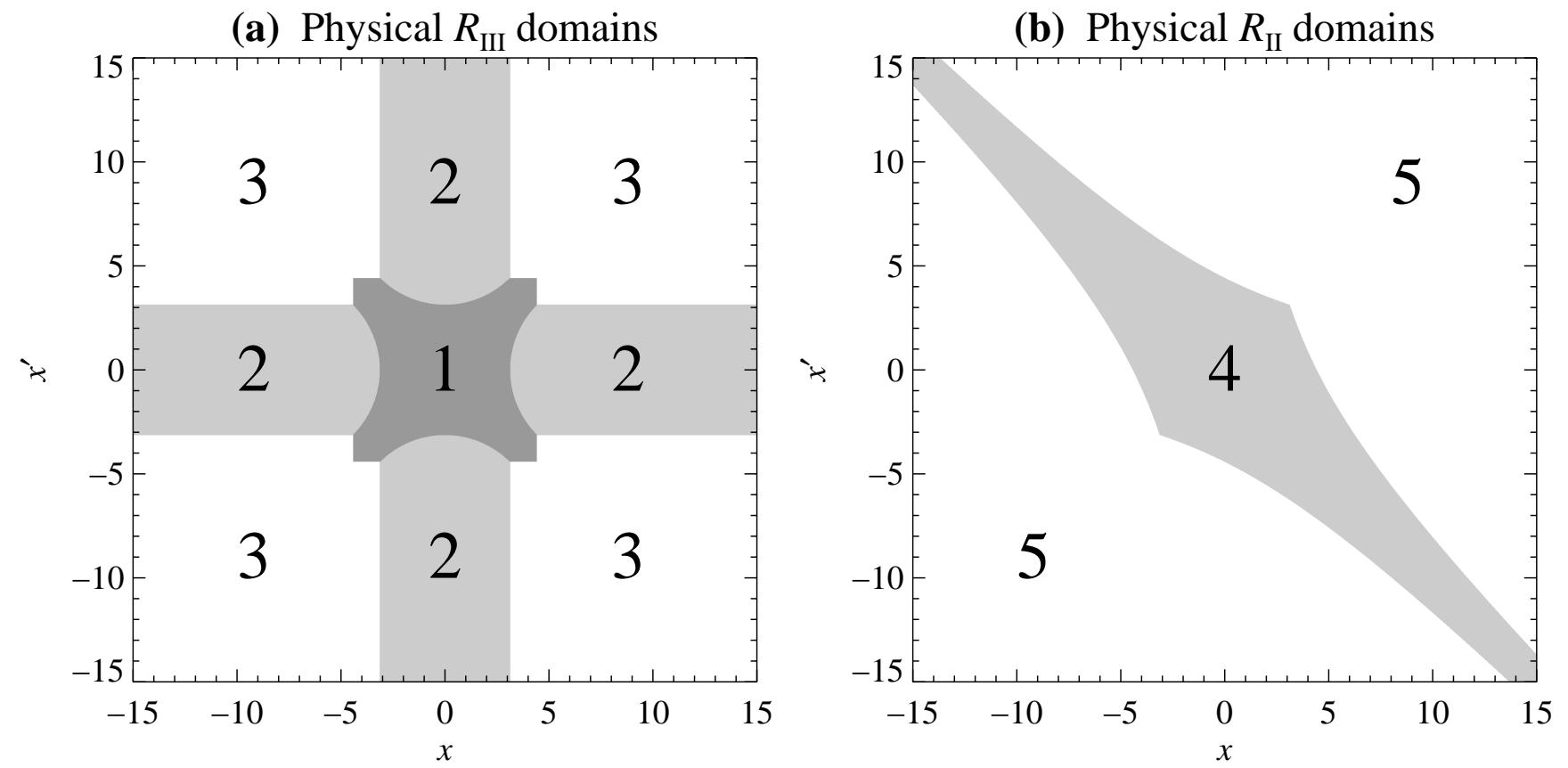

Fig. 1. The $R_{\mathrm{III}}$ and $R_{\mathrm{II}}$ physical domains obtained according to the logical sequence presented in Eqs. (5)-(7) for a Voigt damping parameter $a=10^{-3}$. The corresponding value of the cut-off parameter is $v_{\mathrm{c}}(a) \approx 3.1$.

The physical domains, shown in Fig. 1 for a damping parameter $a=10^{-3}$, can be understood as a sort of recipe. For a given pair of incoming frequency $x^{\prime}$ and outgoing frequency $x$ the plots in Fig. 1, or the logical sequence of Eqs. (5)-(7), determine which expression of the redistribution matrix has to be employed. The redistribution matrices are listed at the end of this section.

Notice that the physical frequency domains always exhibit the same structure or pattern as in Fig. 1 for any value of the damping parameter $a$. The only difference is that the size of the physical domains 1 and 4 and the width of the physical domain 2 scale with the cut-off parameter $v_{\mathrm{c}}(a)$.

\section{Definitions}

Before we can write down the redistribution matrix we have to introduce some notation and definitions. As in Bommier (1997b), we use the collisional parameters (branching ratios)

$\alpha=\frac{\Gamma_{\mathrm{R}}}{\Gamma_{\mathrm{R}}+\Gamma_{\mathrm{I}}+\Gamma_{\mathrm{E}}}$,

$\beta^{(K)}=\frac{\Gamma_{\mathrm{R}}}{\Gamma_{\mathrm{R}}+\Gamma_{\mathrm{I}}+D^{(K)}}$,

and the magnetic field strength parameters

$\Gamma_{B}=g_{J^{\prime}} \frac{\omega_{\mathrm{L}}}{\Gamma_{\mathrm{R}}}$,

$\Gamma_{K}^{\prime}=\beta^{(K)} \Gamma_{B}$,

$\Gamma^{\prime \prime}=\alpha \Gamma_{B}$.

The quantity $g_{J^{\prime}}$ is the statistical weight and $\omega_{\mathrm{L}}$ the Larmor frequency of the upper level. Further, $\left(\Gamma_{R}, \Gamma_{I}, \Gamma_{E}\right)$ represent the radiative de-excitation rate, the inelastic collision rate, and the elastic collision rate respectively. The quantity $D^{(K)}$ is the
$2 K$-multipole destruction rate due to elastic collisions, and is related to $\Gamma_{\mathrm{E}}$ in the form

$D^{(0)}=0$

$D^{(2)}=c \Gamma_{\mathrm{E}}, \quad 0 \leq c \leq 1$.

The $D^{(1)}$ parameter enters the phase matrix components for Stokes $V$, and is not relevant for the present problem.

For the phase matrices we introduce the notation

$\hat{\boldsymbol{P}}_{\mathrm{H}}\left(\boldsymbol{n}, \boldsymbol{n}^{\prime}, \gamma, W_{\mathrm{eff}}\right)=\hat{\boldsymbol{P}}^{(0)}\left(\boldsymbol{n}, \boldsymbol{n}^{\prime}\right)+W_{\mathrm{eff}} \hat{\boldsymbol{P}}_{\mathrm{H}}^{(2)}\left(\boldsymbol{n}, \boldsymbol{n}^{\prime}, \gamma\right)$,

$\hat{\boldsymbol{P}}_{\mathrm{R}}\left(\boldsymbol{n}, \boldsymbol{n}^{\prime}, W_{\mathrm{eff}}\right)=\hat{\boldsymbol{P}}^{(0)}\left(\boldsymbol{n}, \boldsymbol{n}^{\prime}\right)+W_{\mathrm{eff}} \hat{\boldsymbol{P}}_{\mathrm{R}}^{(2)}\left(\boldsymbol{n}, \boldsymbol{n}^{\prime}\right)$,

where the subscript " $H$ " stands for the Hanle phase matrix and the subscript " $R$ " for Rayleigh phase matrix. By setting the effective depolarization parameter $W_{\text {eff }}=W_{2}$ we obtain from Eqs. (15) and (16) the well-known Hanle and Rayleigh phase matrices (Landi Degl'Innocenti \& Landi Degl'Innocenti 1988). The depolarization factor $W_{2}$ depends only on the total angular momentum quantum numbers $J$ and $J^{\prime}$ of the lower and upper level and a listing of $W_{2}$ can be found for various electricdipole transitions in Landi Degl'Innocenti (1984). Note that the terms corresponding to $K=1$ appear only in the equations for Stokes $V$ which is not regarded here.

\section{Redistribution matrices}

With the above definitions at hand we can now write the expressions for the redistribution matrices in the five physical frequency domains. Notice that these expressions have been given by Bommier (1997b). Here we rearrange the different terms to accommodate the definitions (15) and (16), which is necessary for the numerical method presented in this paper. 
The $\hat{\mathscr{R}}_{\text {III }}$ redistribution matrix takes the following form in the physical frequency domains 1 to 3 :

- in domain 1 (ref: Eq. (107) of Bommier 1997b)

$$
\begin{aligned}
\hat{\mathscr{R}}_{\mathrm{II}}( & \left.x, x^{\prime}, \boldsymbol{n}, \boldsymbol{n}^{\prime}, \boldsymbol{B}\right)=R_{\mathrm{III}}\left(x, x^{\prime}\right) \\
& \cdot\left[\beta^{(0)} \hat{\boldsymbol{P}}_{\mathrm{H}}\left(\boldsymbol{n}, \boldsymbol{n}^{\prime}, \Gamma_{2}^{\prime}, W_{\mathrm{eff}}=\frac{\beta^{(2)}}{\beta^{(0)}} W_{2}\right)\right. \\
& \left.-\alpha \hat{\boldsymbol{P}}_{\mathrm{H}}\left(\boldsymbol{n}, \boldsymbol{n}^{\prime}, \Gamma^{\prime \prime}, W_{\mathrm{eff}}=W_{2}\right)\right],
\end{aligned}
$$

- in domain 2 (ref: Eq. (109) of Bommier 1997b)

$$
\begin{gathered}
\hat{\mathscr{R}}_{\mathrm{III}}\left(x, x^{\prime}, \boldsymbol{n}, \boldsymbol{n}^{\prime}, \boldsymbol{B}\right)=R_{\mathrm{III}}\left(x, x^{\prime}\right) \cdot\left(\beta^{(0)}-\alpha\right) \\
\cdot \hat{\boldsymbol{P}}_{\mathrm{H}}\left(\boldsymbol{n}, \boldsymbol{n}^{\prime}, \Gamma_{2}^{\prime}, W_{\mathrm{eff}}=\frac{\beta^{(2)}-\alpha}{\beta^{(0)}-\alpha} W_{2}\right),
\end{gathered}
$$

- in domain 3 (ref: Eq. (110) of Bommier 1997b)

$$
\begin{aligned}
& \hat{\mathscr{R}}_{\mathrm{III}}\left(x, x^{\prime}, \boldsymbol{n}, \boldsymbol{n}^{\prime}, \boldsymbol{B}\right)=R_{\mathrm{III}}\left(x, x^{\prime}\right) \\
& \cdot\left[\frac{\left(\beta^{(0)}-\alpha\right)^{2}}{\beta^{(0)}} \hat{\boldsymbol{P}}_{\mathrm{H}}\left(\boldsymbol{n}, \boldsymbol{n}^{\prime}, \Gamma_{2}^{\prime}, W_{\mathrm{eff}}=\frac{\beta^{(0)}\left(\beta^{(2)}-\alpha\right)^{2}}{\beta^{(2)}\left(\beta^{(0)}-\alpha\right)^{2}} W_{2}\right)\right. \\
& \left.+\frac{\alpha\left(\beta^{(0)}-\alpha\right)}{\beta^{(0)}} \hat{\boldsymbol{P}}_{\mathrm{R}}\left(\boldsymbol{n}, \boldsymbol{n}^{\prime}, W_{\mathrm{eff}}=\frac{\beta^{(0)}\left(\beta^{(2)}-\alpha\right)}{\beta^{(2)}\left(\beta^{(0)}-\alpha\right)} W_{2}\right)\right] .
\end{aligned}
$$

The $\hat{\mathscr{R}}_{\text {II }}$ redistribution matrix has the following form in the physical frequency domains 4 and 5:

- in domain 4 (ref: Eq. (112) of Bommier 1997b)

$$
\begin{aligned}
& \hat{\mathscr{R}}_{\mathrm{II}}\left(x, x^{\prime}, \boldsymbol{n}, \boldsymbol{n}^{\prime}, \boldsymbol{B}\right)= \\
& R_{\mathrm{II}}\left(x, x^{\prime}\right) \cdot \alpha \hat{\boldsymbol{P}}_{\mathrm{H}}\left(\boldsymbol{n}, \boldsymbol{n}^{\prime}, \Gamma^{\prime \prime}, W_{\mathrm{eff}}=W_{2}\right),
\end{aligned}
$$

- in domain 5 (ref: Eq. (113) of Bommier 1997b)

$$
\begin{aligned}
& \hat{\mathscr{R}}_{\mathrm{II}}\left(x, x^{\prime}, \boldsymbol{n}, \boldsymbol{n}^{\prime}, \boldsymbol{B}\right)= \\
& R_{\mathrm{II}}\left(x, x^{\prime}\right) \cdot \alpha \hat{\boldsymbol{P}}_{\mathrm{R}}\left(\boldsymbol{n}, \boldsymbol{n}^{\prime}, W_{\mathrm{eff}}=W_{2}\right) .
\end{aligned}
$$

\subsection{Irreducible transfer equation in Fourier space}

It is possible to simplify Eq. (1) by expanding $\mathscr{I}$ and $\mathscr{S}_{\ell}$ in Fourier series with respect to the azimuth angle $\varphi$. As described in Faurobert-Scholl (1991) and in Nagendra et al. (1998) both intensity and source vector can then be written as 6-component irreducible vectors $I$ and $S$ which satisfy the transfer equation

$\mu \frac{\partial \boldsymbol{I}(\tau, x, \mu)}{\partial \tau}=(\phi(\tau, x)+\beta(\tau))[\boldsymbol{I}(\tau, x, \mu)-\boldsymbol{S}(\tau, x)]$.

For the brevity of notation, we specify the functional dependences from now on only when necessary and as subscripts. In this 6-component version $I$ no longer depends on the azimuth angle $\varphi$ while $S$ depends neither on $\varphi$ nor on $\mu$. Basically, this irreducible transfer equation is solved in the Fourier space, and at the end the intensity $\boldsymbol{I}$ is transformed to the real space to obtain $(I, Q, U)^{\mathrm{T}}$. The total source vector in the 6-component representation is written analogous to Eq. (2) as

$\boldsymbol{S}_{x}=\frac{\phi_{x} \boldsymbol{S}_{\ell, x}+\beta \boldsymbol{B}_{\mathrm{th}}}{\phi_{x}+\beta}$ where $\boldsymbol{B}_{\text {th }}=\left(B_{v_{0}}, 0,0,0,0,0\right)^{\mathrm{T}}$. The 6-component line source vector takes the form

$\boldsymbol{S}_{\ell, x}=\frac{1}{\phi_{x}} \int_{-\infty}^{+\infty}\left(\hat{\boldsymbol{M}}_{\mathrm{II}} R_{\mathrm{II}}+\hat{\boldsymbol{M}}_{\mathrm{III}} R_{\mathrm{III}}\right) \boldsymbol{J}_{x^{\prime}} \mathrm{d} x^{\prime}+\epsilon \boldsymbol{B}_{\mathrm{th}}$.

The $(6 \times 6)$-matrices $\hat{\boldsymbol{M}}_{\mathrm{III}}$ and $\hat{\boldsymbol{M}}_{\mathrm{II}}$ in the Fourier space are abbreviations for

$\hat{\boldsymbol{M}}_{\mathrm{III}}=b_{\mathrm{III}, 1} \hat{\boldsymbol{W}}_{\mathrm{III}, 1}^{2} \hat{\boldsymbol{H}}_{B, \mathrm{III}, 1}+b_{\mathrm{III}, 2} \hat{\boldsymbol{W}}_{\mathrm{III}, 2}^{2} \hat{\boldsymbol{H}}_{B, \mathrm{III}, 2}$

$\hat{\boldsymbol{M}}_{\mathrm{II}}=b_{\mathrm{II}} \hat{\boldsymbol{W}}_{\mathrm{II}}^{2} \hat{\boldsymbol{H}}_{B, \mathrm{II}}$,

where the $b$ 's are scalar factors defined in Sect. 2.4.2, and $\hat{W}$ and $\hat{\boldsymbol{H}}_{B}$ are $(6 \times 6)$-matrices defined in Sect. 2.4.3 and in Sect. 2.4.4 respectively. The mean intensity vector $\boldsymbol{J}_{x^{\prime}}$ is given by

$\boldsymbol{J}_{x^{\prime}}=\frac{1}{2} \int_{-1}^{+1} \hat{\boldsymbol{B}}_{0}^{\mathrm{T}}\left(\mu^{\prime}\right) \hat{\boldsymbol{B}}_{0}\left(\mu^{\prime}\right) \boldsymbol{I}\left(\tau, x^{\prime}, \mu^{\prime}\right) \mathrm{d} \mu^{\prime}$,

where "T" stands for the transpose of matrix $\hat{\boldsymbol{B}}_{0}$. The $(14 \times 6)$-matrix $\hat{\boldsymbol{B}}_{0}$ is defined in the following Sect. 2.4.1.

\subsubsection{The matrix $\hat{\boldsymbol{B}}_{0}$}

$\hat{\boldsymbol{B}}_{0}$ is a $(14 \times 6)$-matrix which may be written in symbolic notation as

$\hat{\boldsymbol{B}}_{0}(\mu)=\left(\begin{array}{cccccc}\boldsymbol{Z}_{0} & \boldsymbol{Z}_{1} & 0 & 0 & 0 & 0 \\ 0 & 0 & \boldsymbol{Z}_{2} & \boldsymbol{Z}_{4} & 0 & 0 \\ 0 & 0 & -\boldsymbol{Z}_{4} & \boldsymbol{Z}_{2} & 0 & 0 \\ 0 & 0 & 0 & 0 & \boldsymbol{Z}_{3} & \boldsymbol{Z}_{5} \\ 0 & 0 & 0 & 0 & \boldsymbol{Z}_{5} & -\boldsymbol{Z}_{3}\end{array}\right)$.

Here the $\boldsymbol{Z}_{i}$ are three-component column vectors, except for $\boldsymbol{Z}_{0}$ and $\boldsymbol{Z}_{1}$ which are two-component vectors (see Eqs. (10)-(13) in Paper III). Note however, that the definition of the $\boldsymbol{Z}_{i}$ vectors differ slightly from their definition given in Paper III. Here the $W$ 's are not included in the expressions for the $\boldsymbol{Z}_{i}$ vectors because they enter the definition of the $\hat{\boldsymbol{M}}$ matrices independently. To distinguish the $\hat{\boldsymbol{B}}$ matrix in this paper from its definition in Paper III we have added the index "0" in Eq. (28).

\subsubsection{Branching factors $b$}

The b's in Eqs. (25) and (26) act as branching ratios. They are functions of the collisional parameters defined in Eqs. (8) and (9) but depend on the physical frequency domains in the form of step functions and are given by

$b_{\mathrm{III}, 1}=\left\{\begin{array}{ll}\beta^{(0)} & , \text { domain 1 } \\ \beta^{(0)} & \text {, domain 2 } \\ \frac{\left(\beta^{(0)}-\alpha\right)^{2}}{\beta^{(0)}} & , \text { domain 3 }\end{array}\right.$,

$b_{\text {III, } 2}=\left\{\begin{array}{ll}-\alpha & , \text { domain 1 } \\ -\alpha & \text {, domain 2 } \\ \frac{\alpha\left(\beta^{(0)}-\alpha\right)}{\beta^{(0)}} & , \text { domain 3 }\end{array}\right.$,

$b_{\mathrm{II}}= \begin{cases}\alpha, & \text { domain } 4 \\ \alpha & , \text { domain } 5\end{cases}$ 


\subsubsection{The depolarization matrices $\hat{W}$}

The $(6 \times 6) \hat{\boldsymbol{W}}$-matrices contain the $W_{2}$ as well as the collisional parameters $\alpha, \beta^{(0)}$, and $\beta^{(2)}$ and act as depolarization matrices. They have a general, diagonal form

$\hat{\boldsymbol{W}}=\operatorname{diag}\left\{1, \sqrt{W_{\text {eff }}}, \sqrt{W_{\text {eff }}}, \sqrt{W_{\text {eff }}}, \sqrt{W_{\text {eff }}}, \sqrt{W_{\text {eff }}}\right\}$,

in terms of the effective depolarization factors $W_{\text {eff }}$. The factors $W_{\text {eff }}$ to be used in the depolarization matrices $\hat{W}$ appearing in Eqs. (25)-(26) are defined as

$\hat{W}_{\mathrm{III}, 1}: \begin{cases}W_{\mathrm{eff}}=\frac{\beta^{(2)}}{\beta^{(0)}} W_{2} & , \text { domain } 1 \\ W_{\mathrm{eff}}=\frac{\beta^{(2)}-\alpha}{\beta^{(0)}-\alpha} W_{2} & , \text { domain 2 } \\ W_{\mathrm{eff}}=\frac{\beta^{(0)}\left(\beta^{(2)}-\alpha\right)^{2}}{\beta^{(2)}\left(\beta^{(0)}-\alpha\right)^{2}} W_{2} & , \text { domain 3 }\end{cases}$

$\hat{W}_{\mathrm{III}, 2}:\left\{\begin{array}{ll}W_{\mathrm{eff}}=W_{2} & , \text { domain } 1 \\ W_{\mathrm{eff}}=\frac{\beta^{(2)}-\alpha}{\beta^{(0)}-\alpha} W_{2} & , \text { domain 2 } \\ W_{\mathrm{eff}}=\frac{\beta^{(0)}\left(\beta^{(2)}-\alpha\right)}{\beta^{(2)}\left(\beta^{(0)}-\alpha\right)} W_{2} & , \text { domain 3 }\end{array}\right.$,

$\hat{\boldsymbol{W}}_{\mathrm{II}}:\left\{\begin{array}{l}W_{\mathrm{eff}}=W_{2}, \text { domain } 4 \\ W_{\text {eff }}=W_{2}, \text { domain } 5\end{array}\right.$.

\subsubsection{The magnetic matrices $\hat{\boldsymbol{H}}_{B}$}

The $\hat{\boldsymbol{H}}_{B}$-matrices in Eqs. (25)-(26) have exactly the same form as defined in Paper III and are thus not explicitly given here. The only difference is that now different Hanle parameters $\gamma$ should be used for different physical domains in the $\left(x, x^{\prime}\right)$-plane. These $\hat{\boldsymbol{H}}_{B}$-matrices are given by

$\hat{\boldsymbol{H}}_{B, \mathrm{III}, 1}=\hat{\boldsymbol{H}}_{B}\left(\theta_{B}, \varphi_{B}, \gamma\right)$ with $\left\{\begin{array}{l}\gamma=\Gamma_{2}^{\prime}, \text { domain } 1 \\ \gamma=\Gamma_{2}^{\prime}, \text { domain } 2 \\ \gamma=\Gamma_{2}^{\prime}, \text { domain 3 }\end{array}\right.$,

$\hat{\boldsymbol{H}}_{B, \mathrm{III}, 2}=\hat{\boldsymbol{H}}_{B}\left(\theta_{B}, \varphi_{B}, \gamma\right)$ with $\left\{\begin{array}{l}\gamma=\Gamma^{\prime \prime}, \text { domain } 1 \\ \gamma=\Gamma_{2}^{\prime}, \text { domain } 2 \\ \gamma=0, \text { domain 3 }\end{array}\right.$,

$\hat{\boldsymbol{H}}_{B, \mathrm{II}}=\hat{\boldsymbol{H}}_{B}\left(\theta_{B}, \varphi_{B}, \gamma\right)$ with $\left\{\begin{array}{ll}\gamma=\Gamma^{\prime \prime} & \text {, domain 4 } \\ \gamma=0 & \text {, domain 5 }\end{array}\right.$,

where $\hat{\boldsymbol{H}}_{B}\left(\theta_{B}, \varphi_{B}, \gamma\right)$ is the magnetic matrix defined by Eq. (48) in Paper III.

\subsection{Discussion on the irreducible transfer equation}

The 6-component irreducible transfer equation has to be derived via a 14-component representation, which is omitted here, but the details can be found in Paper III. However, some additional comments are important for the clarification of the method presented in this paper.

A necessary condition for the derivation of the irreducible 6-component representation of the transfer equation is, that the 14-component total source vector $S_{\mathrm{F}}$ (using the index "F" to indicate 14-component quantities as in Paper III) can be factorized in the form

$S_{\mathrm{F}}=\hat{\boldsymbol{B}}_{0} S$.

In the formalism introduced here, we are dealing with effective depolarization factors $W_{\text {eff }}$ that are different in different physical frequency domains. Thus we are forced to introduce the matrix $\hat{\boldsymbol{B}}_{0}$. If, on the other hand, we preserve the original definition of $\hat{\boldsymbol{B}}$ from Paper III, which includes the factor $W$ or $W_{\text {eff }}$ respectively, then it would not be possible to factorize out a common $\hat{\boldsymbol{B}}$-matrix, and condition (39) would not be fulfilled. As a result of the introduction of $\hat{\boldsymbol{B}}_{0}$ the transformation of 6-component quantities to real space 3-component quantities has to be slightly adapted. It is now done using Eqs. (81)-(83) of Paper III, where $W$ is set to one, irrespective of the actual value of $W_{2}$.

Note that the $\hat{\boldsymbol{W}}$-matrices commute with the $\hat{\boldsymbol{H}}_{B}$-matrices due to the special block diagonal structure of $\hat{\boldsymbol{H}}_{B}$. However, they do not commute with $\hat{\boldsymbol{B}}_{0}^{\mathrm{T}} \hat{\boldsymbol{B}}_{0}$. Note further that the product

$\hat{\boldsymbol{B}}=\hat{\boldsymbol{B}}_{0} \hat{\boldsymbol{W}}$,

gives the relation between the $\hat{\boldsymbol{B}}$-matrix as defined in Paper III and the corresponding $\hat{\boldsymbol{B}}_{0}$-matrix introduced in this paper.

It is crucial to observe that the branching factors $b$, and the matrices $\hat{\boldsymbol{W}}$ and $\hat{\boldsymbol{H}}_{B}$ remain constant within a given physical frequency domain and hence depend only on depth via the depth dependence of the collisional transition rates and of the magnetic field. However, in general their values are different in different physical frequency domains, which makes these quantities apparently "frequency dependent" in a stepwise manner. Thus it is not possible to take them outside the frequency integral in Eq. (24). This point has to be considered carefully in the numerical method of solution.

\section{The numerical method of solution}

\subsection{The generalized PALI method}

In this section we give a few basic equations which indicate a generalization of the PALI-PRD method (Paper IV) to the more general problem discussed here. It is based on the CRDCS method of Paletou \& Auer (1995). The formal solution of the Hanle transfer equation may be stated in terms of the full Lambda operator as

$\boldsymbol{J}_{x}=\hat{\boldsymbol{\Lambda}}_{x}\left[\boldsymbol{S}_{x}\right]$,

where $\hat{\boldsymbol{\Lambda}}_{x}$ operates on the quantity within [ ]. By defining a local, monochromatic approximate Lambda operator $\hat{\boldsymbol{\Lambda}}_{x}^{*}$ as

$\hat{\boldsymbol{\Lambda}}_{x}=\hat{\boldsymbol{\Lambda}}_{x}^{*}+\delta \hat{\boldsymbol{\Lambda}}_{x}=\hat{\boldsymbol{\Lambda}}_{x}^{*}+\left(\hat{\boldsymbol{\Lambda}}_{x}-\hat{\boldsymbol{\Lambda}}_{x}^{*}\right)$,

we can set up an iterative scheme to compute the source vectors, namely

$\boldsymbol{S}_{x}^{(n+1)}=\boldsymbol{S}_{x}^{(n)}+\delta \boldsymbol{S}_{x}^{(n)}$,

$\boldsymbol{S}_{\ell, x}^{(n+1)}=\boldsymbol{S}_{\ell, x}^{(n)}+\delta \boldsymbol{S}_{\ell, x}^{(n)}$ 
where the superscript $(n)$ refers to the $n$th iteration step. From Eqs. (42) and (43) it follows by keeping only terms up to first order, that

$\boldsymbol{J}_{x}^{(n+1)} \approx \boldsymbol{J}_{x}^{(n)}+\hat{\boldsymbol{\Lambda}}_{x}^{*}\left[\delta \boldsymbol{S}_{x}^{(n)}\right]$.

From Eq. (23) we find

$\delta \boldsymbol{S}_{x}^{(n)}=p_{x} \delta \boldsymbol{S}_{\ell, x}^{(n)}$,

with

$p_{x}=\frac{\phi_{x}}{\phi_{x}+\beta}$.

Further, note that

$\hat{\boldsymbol{\Lambda}}_{x}^{*}\left[p_{x} \delta \boldsymbol{S}_{\ell, x}\right]=p_{x} \hat{\boldsymbol{\Lambda}}_{x}^{*}\left[\delta \boldsymbol{S}_{\ell, x}\right]$,

since $p_{x}$ is a scalar quantity, and $\hat{\boldsymbol{\Lambda}}_{x}^{*}$ is a linear operator. Inserting Eqs. (24), (45), (46) and (48) into Eq. (44) we find the equation for the corrections to the line source vector $\delta \boldsymbol{S}_{\ell, x}^{(n)}$

$\delta S_{\ell, x}^{(n)}-\frac{1}{\phi_{x}} \int_{-\infty}^{+\infty}\left(\hat{\boldsymbol{M}}_{\mathrm{II}} R_{\mathrm{II}}+\hat{\boldsymbol{M}}_{\mathrm{III}} R_{\mathrm{III}}\right)$

$\times p_{x^{\prime}} \hat{\boldsymbol{\Lambda}}_{x^{\prime}}^{*}\left[\delta \boldsymbol{S}_{\ell, x^{\prime}}^{(n)}\right] \mathrm{d} x^{\prime}=\boldsymbol{r}_{x}^{(n)}$

with the frequency dependent residual vector given by

$\boldsymbol{r}_{x}^{(n)}=\boldsymbol{S}_{\mathrm{FS}, \ell, x}^{(n)}-\boldsymbol{S}_{\ell, x}^{(n)}$.

The formal line source vector $S_{\mathrm{FS}, \ell, x}^{(n)}$ is obtained from

$\boldsymbol{S}_{\mathrm{FS}, \ell, x}^{(n)}=\frac{1}{\phi_{x}} \int_{-\infty}^{+\infty}\left(\hat{\boldsymbol{M}}_{\mathrm{II}} R_{\mathrm{II}}+\hat{\boldsymbol{M}}_{\mathrm{III}} R_{\mathrm{III}}\right) \boldsymbol{J}_{x^{\prime}}^{(n)} \mathrm{d} x^{\prime}+\epsilon \boldsymbol{B}_{\mathrm{th}}$,

where the mean intensity $\boldsymbol{J}_{x}^{(n)}=\hat{\boldsymbol{\Lambda}}_{x}\left[\boldsymbol{S}_{x}^{(n)}\right]$ is computed with the short characteristic formal solver (FS).

\section{Iteration algorithm}

The algorithm to solve the polarized line transfer Eq. (22) by the PALI method can now be summarized as:

Step 1: Calculation of the approximate local operator $\hat{\Lambda}_{x}^{*}$.

Step 2: Defining an initial source vector to initiate the iterative procedure.

Step 3: Calculation of the mean intensity vector $\boldsymbol{J}_{x}^{(n)}$ and of the line source vector $S_{\mathrm{FS}, \ell, x}^{(n)}$ through an accurate Formal Solver (FS).

Step 4: Solving Eq. (49) to estimate the source vector corrections $\delta \boldsymbol{S}_{\ell, x}^{(n)}$ (see also Sect. 3.2).

Step 5: Updating the line source vector $\boldsymbol{S}_{\ell, x}$ and the total source vector $\boldsymbol{S}_{x}$.

Step 6: Testing for convergence. If no convergence, repeat steps 3 to 6 . If convergence, then end the iterative sequence.

It is observed that the iterative procedure exhibits a uniform convergence even in difficult cases. The well-known acceleration procedures like $\mathrm{Ng}$ acceleration perform very well. These aspects are discussed already in previous papers on PALI.

The details of step 4 will be addressed in Sect. 3.2.

\subsection{Estimation of source vector corrections}

The numerical method to perform step no. 4 of the iteration algorithm given above, namely the method to solve Eq. (49) for the source vector corrections, will be explained in more detail in this section. We employ the so called CRD-CS scheme developed by Paletou \& Auer (1995) which has subsequently been generalized to polarized line transfer and implemented into the PALI method in Paper II. It proved to be very efficient since the system of Eqs. (49) gets decoupled such that $\delta \boldsymbol{S}_{\ell, x}^{(n)}$ is obtained through simple manipulations.

Since we are dealing here only with the corrections of the source vector it is possible to apply further approximations in this step as long as we remain close enough to the true physics. In step 4 of the iteration algorithm we have to solve Eq. (49). The idea is to simplify and approximate Eq. (49) such that its solution becomes much easier and faster. Thus we only obtain an approximation of the source vector correction. This does not matter a lot because the source vector will be corrected further in the following iteration steps. It turns out that this procedure speeds up the iteration steps considerably and proves to be very robust and stable as long as the main physics is still contained in the simplified version of Eq. (49).

First we introduce approximated frequency domains in Sect. 3.2.1. Then we approximate the integrals in Eq. (49) involving $R_{\mathrm{III}}$ and $R_{\mathrm{II}}$ in Sects. 3.2.2 and 3.2.3 respectively and introduce the necessary notation. Later, in Sects. 3.2.4 and 3.2.5, we combine these simplifications and write the approximated versions of Eq. (49) for the source vector corrections $\delta \boldsymbol{S}_{\ell, x}^{(n)}$.

\subsubsection{Approximated domains}

Only if the frequency domains are redefined as shown in Fig. 2 does it become possible to apply the core-wing separation scheme. As will become clear below, it is necessary to keep the boundaries as straight vertical and horizontal lines (Fig. 2). The new approximated domains are marked with an asterisk to distinguish them clearly from the actual physical domains shown in Fig. 1.

Lest there be a confusion, we would like to emphasize that the physical frequency domains, which are shown in Fig. 1 are actually employed in the evaluation of scattering integrals and mean intensity computations in step 3 of the iteration algorithm. The "approximated domains" which are discussed in this section are required only for the purpose of computing the source vector corrections in the PALI iterations. Therefore, the final result of the iteration procedure is consistent with the physical domains.

In the following, we outline the CRD-CS method that allows us to treat core and wing corrections separately. The redistribution function $R_{\mathrm{III}}$ is approximated as CRD and the wing part of integrals involving $R_{\mathrm{III}}$ is neglected. The function $R_{\mathrm{II}}$ is treated as CRD in the line core and as coherent scattering in the wings. Thus core and wings decouple from each other. The crucial point is that the CRD integral over the line core, which will be defined as $\Delta \boldsymbol{T}_{\text {core }}^{(n)}$ in Eq. (55), is independent of the frequency $x$. This decouples the equations for different values of $x$ in expression (49). 
(a) Approximated core-wing $\mathbf{R}_{\mathrm{III}}{ }^{- \text {domains }}$

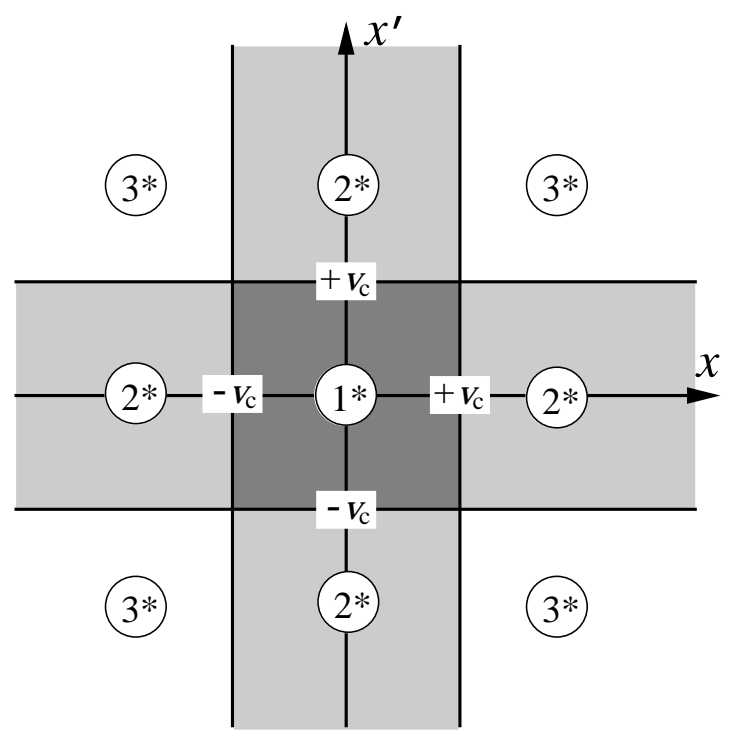

(b) Approximated core-wing $\mathbf{R}_{I^{-}}$-domains

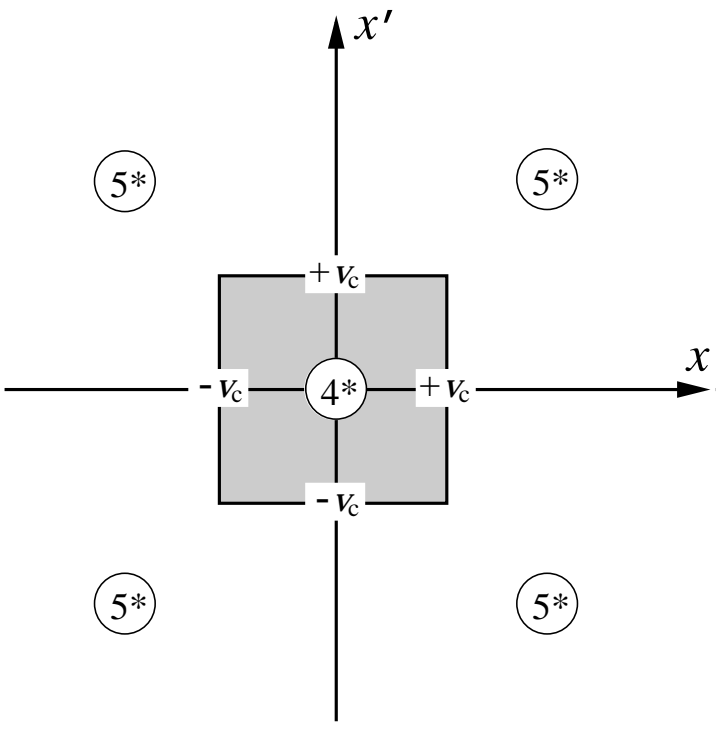

Fig. 2. The domains of $R_{\mathrm{III}}$ (panel a)) and $R_{\mathrm{II}}$ integrals (panel b)) are approximated as shown for solving Eq. (49) with the generalized core-wing scheme. The numbers of the approximated domains are marked with an asterisk to distinguish them from the physical domains.

\subsubsection{Integrals involving $R_{\mathrm{III}}$}

In the computation of the source vector corrections, $R_{\mathrm{III}}$ is approximated by CRD, namely, we assume $R_{\mathrm{III}} \approx \phi_{x} \phi_{x^{\prime}}$. Notice again, that this approximation is made only to solve Eq. (49). In the same spirit, the wing part of the frequency integral involving $R_{\mathrm{III}}$ is neglected which is possible because $\phi_{x}$ is sufficiently small in the wings. Thus the $R_{\text {III }}$ part of the integral in Eq. (49) simplifies to

$\frac{1}{\phi_{x}} \int_{-\infty}^{+\infty} \hat{\boldsymbol{M}}_{\mathrm{III}} R_{\mathrm{III}} p_{x^{\prime}} \hat{\boldsymbol{\Lambda}}_{x^{\prime}}^{*}\left[\delta \boldsymbol{S}_{\ell, x^{\prime}}^{(n)}\right] \mathrm{d} x^{\prime} \approx\left\{\begin{array}{l}\hat{\boldsymbol{M}}_{\mathrm{D} 1} \Delta \boldsymbol{T}_{\mathrm{core}}^{(n)},|x| \leq v_{\mathrm{c}}(a) \\ \hat{\boldsymbol{M}}_{\mathrm{D} 2} \Delta \boldsymbol{T}_{\mathrm{core}}^{(n)},|x|>v_{\mathrm{c}}(a)\end{array}\right.$,

with the following definitions

$\hat{\boldsymbol{M}}_{\mathrm{D} 1}=\left.\hat{\boldsymbol{M}}_{\mathrm{III}}\right|_{\left(x, x^{\prime}\right) \in \operatorname{domain} 1^{*}}$,

$\hat{\boldsymbol{M}}_{\mathrm{D} 2}=\left.\hat{\boldsymbol{M}}_{\mathrm{III}}\right|_{\left(x, x^{\prime}\right) \in \text { domain } 2^{*}}$,

$\Delta \boldsymbol{T}_{\text {core }}^{(n)}=\int_{-v_{\mathrm{c}}(a)}^{+v_{\mathrm{c}}(a)} \phi_{x^{\prime}} p_{x^{\prime}} \hat{\boldsymbol{\Lambda}}_{x^{\prime}}^{*}\left[\delta \boldsymbol{S}_{\ell, x^{\prime}}^{(n)}\right] \mathrm{d} x^{\prime}$

The matrix $\hat{\boldsymbol{M}}_{\mathrm{D} 3}$, corresponding to the approximated domain $3^{*}$, does not appear in Eq. (52) because the wing part of the $x^{\prime}$-integral is neglected, with the wings defined by $|x|>v_{\mathrm{c}}(a)$.

\subsubsection{Integrals involving $R_{\|}$}

The $R_{\mathrm{II}}$ part of the integral in Eq. (49) is approximated according to the core-wing approach (see Eqs. (16)-(17) in Paper IV):

$$
\int_{-\infty}^{+\infty} \frac{R_{\mathrm{II}}}{\phi_{x}} \boldsymbol{Y} \mathrm{d} x^{\prime} \approx\left(1-\alpha_{x}\right) \int_{-v_{\mathrm{c}}(a)}^{+v_{\mathrm{c}}(a)} \phi_{x^{\prime}} \boldsymbol{Y} \mathrm{d} x^{\prime}+\alpha_{x} \int_{-\infty}^{+\infty} \delta\left(x-x^{\prime}\right) \boldsymbol{Y} \mathrm{d} x^{\prime}
$$

for any vector $\boldsymbol{Y}$. The separation coefficient $\alpha_{x}$ is defined as

$\alpha_{x}=\left\{\begin{array}{ll}0 & ,|x| \leq v_{\mathrm{c}}(a) \\ \frac{R_{\mathrm{II}}\left(x, x^{\prime}\right)}{\phi_{x}}, & ,|x|>v_{\mathrm{c}}(a)\end{array}\right.$.

In the core-wing approach, clearly $R_{\mathrm{II}}$ is approximated by CRD in the line core and as coherent scattering (CS) in the line wings (for this reason it is called CRD-CS scheme in previous papers). Applying such core-wing separation in Eq. (49) we can write

$$
\begin{aligned}
& \int_{-\infty}^{+\infty} \hat{\boldsymbol{M}}_{\mathrm{II}} \frac{R_{\mathrm{II}}}{\phi_{x}} p_{x^{\prime}} \hat{\boldsymbol{\Lambda}}_{x^{\prime}}^{*}\left[\delta \boldsymbol{S}_{\ell, x^{\prime}}^{(n)}\right] \mathrm{d} x^{\prime} \\
& \approx\left\{\begin{array}{l}
\hat{\boldsymbol{M}}_{\mathrm{D} 4} \Delta \boldsymbol{T}_{\mathrm{core}}^{(n)}, \\
\left(1-\alpha_{x}\right) \hat{\boldsymbol{M}}_{\mathrm{D} 5} \Delta \boldsymbol{T}_{\mathrm{core}}^{(n)} \\
+\alpha_{x} \hat{\boldsymbol{M}}_{\mathrm{D} 5} p_{x} \hat{\boldsymbol{\Lambda}}_{x}^{*}\left[\delta \boldsymbol{S}_{\ell, x}^{(n)}\right],|x|>v_{\mathrm{c}}(a)
\end{array}\right.
\end{aligned}
$$

with the following definitions:

$\hat{\boldsymbol{M}}_{\mathrm{D} 4}=\left.\hat{\boldsymbol{M}}_{\mathrm{II}}\right|_{\left(x, x^{\prime}\right) \in \text { domain } 4^{*}}$,

$\hat{\boldsymbol{M}}_{\mathrm{D} 5}=\left.\hat{\boldsymbol{M}}_{\mathrm{II}}\right|_{\left(x, x^{\prime}\right) \in \text { domain } 5^{*}}$.

\subsubsection{Equations for $\delta \boldsymbol{S}_{\ell, x}$ in the line core frequencies}

With the expressions (52) and (58) for the frequency integrals we obtain an approximate equation for the correction of the line source vector $\delta \boldsymbol{S}_{\ell, x}^{(n)}$. For $x$ in the core, that is $|x| \leq v_{\mathrm{c}}(a)$, we obtain, by inserting Eqs. (52) and (58) into Eq. (49)

$\delta \boldsymbol{S}_{\ell, x}^{(n)}=\left(\hat{\boldsymbol{M}}_{\mathrm{D} 1}+\hat{\boldsymbol{M}}_{\mathrm{D} 4}\right) \Delta \boldsymbol{T}_{\mathrm{core}}^{(n)}+\boldsymbol{r}_{x}^{(n)}$.

Applying from the left the integral operator

$\int_{-v_{\mathrm{c}}(a)}^{+v_{\mathrm{c}}(a)} \phi_{x} p_{x} \hat{\mathbf{\Lambda}}_{x}^{*}[\mathrm{]} \mathrm{d} x$ 
on Eq. (61), we get an equation for $\Delta \boldsymbol{T}_{\text {core }}^{(n)}$

$\Delta \boldsymbol{T}_{\mathrm{core}}^{(n)}=\left[\hat{\boldsymbol{I}}-\int_{-v_{\mathrm{c}}(a)}^{+v_{\mathrm{c}}(a)} \phi_{x} p_{x} \hat{\boldsymbol{\Lambda}}_{x}^{*} \mathrm{~d} x \cdot\left(\hat{\boldsymbol{M}}_{\mathrm{D} 1}+\hat{\boldsymbol{M}}_{\mathrm{D} 4}\right)\right]^{-1} \overline{\boldsymbol{r}}_{\text {core }}^{(n)}$

with $\hat{\boldsymbol{I}}$ the $(6 \times 6)$ identity matrix, and with

$$
\overline{\boldsymbol{r}}_{\mathrm{core}}^{(n)}=\int_{-v_{\mathrm{c}}(a)}^{+v_{\mathrm{c}}(a)} \phi_{x} p_{x} \hat{\boldsymbol{\Lambda}}_{x}^{*}\left[\boldsymbol{r}_{x}^{(n)}\right] \mathrm{d} x .
$$

It is important to notice that the operator $\boldsymbol{\Delta} \boldsymbol{T}_{\text {core }}^{(n)}$ is frequency independent. This is made possible through the use of "corewing separation" and later the application of "approximated domains".

\subsubsection{Equations for $\delta \boldsymbol{S}_{\ell, x}$ in the wing frequencies}

We obtain the equation for $\delta \boldsymbol{S}_{\ell, x}^{(n)}$ with $x$ in the wing, namely $|x|>v_{\mathrm{c}}(a)$, by inserting Eqs. (52) and (58) into Eq. (49)

$$
\begin{aligned}
\delta \boldsymbol{S}_{\ell, x}^{(n)}= & {\left[\hat{\boldsymbol{I}}-\alpha_{x} p_{x} \hat{\boldsymbol{M}}_{\mathrm{D} 5} \hat{\boldsymbol{\Lambda}}_{x}^{*}\right]^{-1} } \\
& \cdot\left[\left(\hat{\boldsymbol{M}}_{\mathrm{D} 2}+\left(1-\alpha_{x}\right) \hat{\boldsymbol{M}}_{\mathrm{D} 5}\right) \boldsymbol{\Delta} \boldsymbol{T}_{\mathrm{core}}^{(n)}+\boldsymbol{r}_{x}^{(n)}\right] .
\end{aligned}
$$

For obtaining the correction to the line source vector $\delta \boldsymbol{S}_{\ell, x}^{(n)}$ (step 4 of the iteration algorithm given at the end of Sect. 3.1) we proceed as follows:

Step 4.1: Solve Eq. (63) for the vector $\Delta \boldsymbol{T}_{\text {core }}^{(n)}$.

Step 4.2: Substitute $\Delta \boldsymbol{T}_{\text {core }}^{(n)}$ into Eqs. (61) and (65) to obtain $\delta \boldsymbol{S}_{\ell, x}^{(n)}$.

Now the great advantages of the core-wing separation scheme become apparent. In expression (49), the equations for different frequency points are coupled to each other through the frequency integral. However, it can be noticed that such a coupling does not exist in Eq. (61) and Eq. (65). Thus we need to invert for each frequency point $x$ only a $(6 \times 6)$ system of equations.

A further advantage of the core-wing separation scheme is that the evaluation of the matrices $\hat{\boldsymbol{M}}_{\mathrm{D} 1}, \hat{\boldsymbol{M}}_{\mathrm{D} 2}, \hat{\boldsymbol{M}}_{\mathrm{D} 4}, \hat{\boldsymbol{M}}_{\mathrm{D} 5}$, and the matrix inversions in Eqs. (63) and (65) need to be performed only once before starting the whole iteration sequence, even for a realistic atmosphere. This reduces the CPU time required for each PALI iteration step considerably.

\subsection{Convergence criterion}

The convergence criterion is based on the relative change of the intensity source function as well as on the surface polarization. The iteration is continued as long as

$\max \left\{c_{1}^{(n)}, c_{2}^{(n)}\right\}<c_{\max }$,

where

$$
\begin{aligned}
& c_{1}^{(n)}=\max _{\tau, x, \mu}\left\{\frac{\left|\delta \mathscr{S}_{I}^{(n)}(\tau, x, \mu)\right|}{\left|\mathscr{S}_{I}^{(n)}(\tau, x, \mu)\right|}\right\}, \\
& c_{2}^{(n)}=\max _{x, \mu}\left\{\frac{\left|p^{(n)}(x, \mu)-p^{(n-1)}(x, \mu)\right|}{\left|p^{(n)}(\tau, x, \mu)\right|}\right\},
\end{aligned}
$$

with $p=\sqrt{Q^{2}+U^{2}} / I$ the degree of linear polarization at the surface. If not otherwise stated, we have chosen $c_{\max }=10^{-3}$.

\section{Testing the generalized PALI method}

In this section we will discuss the convergence characteristics of the proposed numerical method and test it by comparing with independent methods.

\subsection{Convergence characteristics}

The convergence behavior of the PALI methods is well studied previously (Papers II and III). Here we would like to test the new method on a typical case as well as on a particularly difficult case with slow convergence.

\section{Model parameters}

For the computation of panel (a) in Fig. 3 we have employed the model parameters $\left(T, a, \epsilon, \beta, \Gamma_{\mathrm{E}} / \Gamma_{\mathrm{R}}\right)=(2 \times$ $\left.10^{6}, 10^{-3}, 10^{-4}, 0,10^{-1}\right)$ with the magnetic field parameters $\left(\Gamma_{B}, \theta_{B}, \varphi_{B}\right)=\left(1,30^{\circ}, 0^{\circ}\right)$. Both at the bottom and the top of the isothermal slab we have employed zero incident radiation as the boundary conditions.

Only one parameter and the boundary conditions have been changed for computing the results shown in panel (b) of Fig. 3. The thermalization parameter $\epsilon$ is set to zero, i.e. we consider a pure scattering medium. The relevance of a $\epsilon=0$ case is discussed in Sect. 4.3. The model parameters in panel (b) are thus $\left(T, a, \epsilon, \beta, \Gamma_{\mathrm{E}} / \Gamma_{\mathrm{R}}\right)=\left(2 \times 10^{6}, 10^{-3}, 0,0,10^{-1}\right)$ with the magnetic field parameters $\left(\Gamma_{B}, \theta_{B}, \varphi_{B}\right)=\left(1,30^{\circ}, 0^{\circ}\right)$. The boundary conditions employed correspond to a pure scattering medium irradiated at $\tau=T$ with an unpolarized radiation field given by

$\boldsymbol{I}(\tau=T, x, \mu)=\boldsymbol{B}_{\mathrm{th}}(\tau=T)$,

and no radiation incident at the upper boundary $\tau=0$.

For both the panels we have employed $W_{2}=1$ and $D^{(2)}=$ $0.5 \Gamma_{\mathrm{E}}$. We have used a logarithmic optical depth $(\tau)$ scale with a resolution of 5 depth points per decade, 5 Gaussian latitude angles $(\theta)$ in $[0<\mu \leq 1]$, and a 71 point non-uniform frequency $(x)$ grid with the last frequency point $x_{\max }=100$. In all computations shown in this paper the Planck function $B_{v_{0}}$ has been set to one.

\section{Discussion}

Figure 3 shows the convergence behavior of the new PALI method for two cases - a typical optically thick model on the left panel (a) and a pure conservative scattering atmospheric model which is known to lead to a slow convergence, on the right panel (b) of the figure. The total source vector at frequency $x=3$ for each iteration step is plotted. The converged solution is marked by dots.

Panel (a) depicts the rapid convergence which is typical of the PALI method. Clearly the rate of convergence is slower in the conservative scattering case shown in panel (b). The grouping of four successive solutions is due to the 4-step marching 
(a) Self-emitting medium
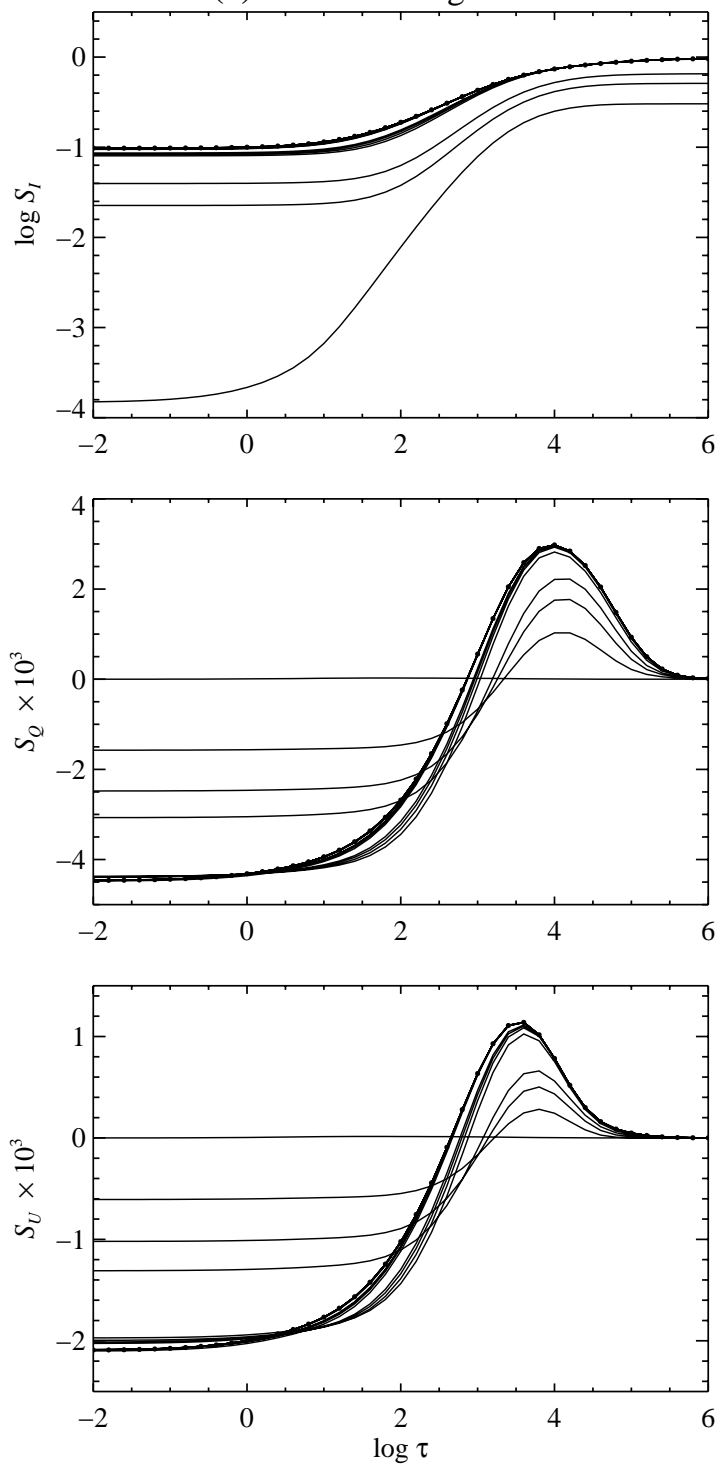

(b) Conservative scattering medium
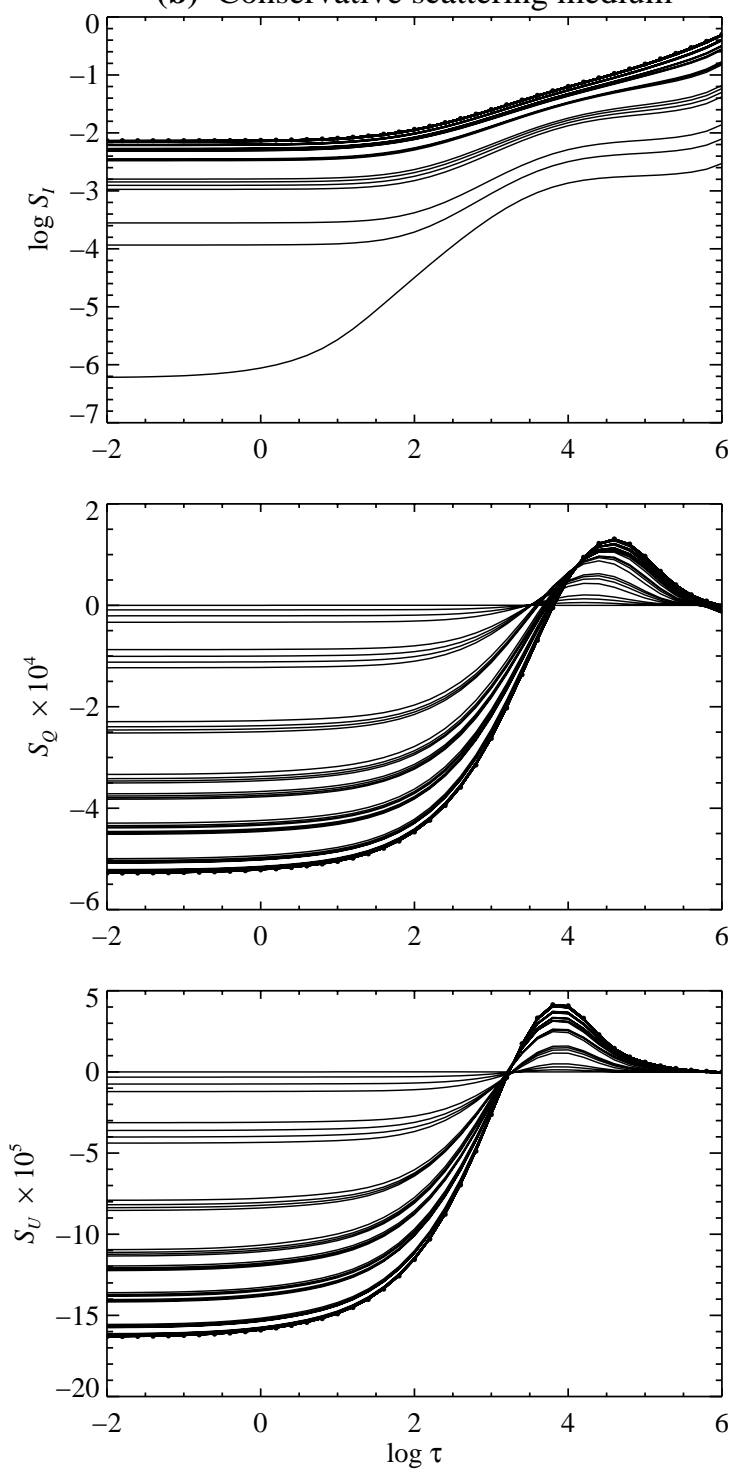

Fig. 3. Convergence behavior of the polarized total source functions for a frequency $x=3$ in the direction $\mu=0.11$ and $\varphi=0^{\circ}$. Panel a): the self-emitting slab model characterized by the parameters $\left(T, a, \epsilon, \beta, \Gamma_{\mathrm{E}} / \Gamma_{\mathrm{R}}\right)=\left(2 \times 10^{6}, 10^{-3}, 10^{-4}, 0,10^{-1}\right)$ and the magnetic field represented by $\left(\Gamma_{B}, \theta_{B}, \varphi_{B}\right)=\left(1,30^{\circ}, 0^{\circ}\right)$. The convergence characteristics are typical of the PALI method. Panel $\left.\mathbf{b}\right)$ : the results for an irradiated pure scattering slab model characterized by the parameters $\left(T, a, \epsilon, \beta, \Gamma_{\mathrm{E}} / \Gamma_{\mathrm{R}}\right)=\left(2 \times 10^{6}, 10^{-3}, 0,0,10^{-1}\right)$ and the magnetic field parameters given by $\left(\Gamma_{B}, \theta_{B}, \varphi_{B}\right)=\left(1,30^{\circ}, 0^{\circ}\right)$. Notice the slow, but uniform convergence in the case of a pure scattering medium. The dots identify the converged solution.

of the $\mathrm{Ng}$-acceleration procedure. Further tests have shown that the convergence of the PALI method becomes slightly slower for the extreme combination of very small $\epsilon$, dominating contribution of $R_{\mathrm{II}}$ redistribution function, and very thick or semiinfinite slab atmospheres. For all other normal and typical combinations of model parameters, PALI exhibits the typical rapid convergence behavior as shown on panel (a) of Fig. 3.

The reason for the slow convergence characteristics for the right hand side panel of Fig. 3 lies in the highly non-local and scattering dominated character of the case. The parameter choice $\epsilon=0$ and $\beta=0$ implies pure scattering without any true absorption and emission. Together with the dominating $R_{\mathrm{II}}$ contribution over that of $R_{\mathrm{III}}$ (because of $\Gamma_{\mathrm{E}} / \Gamma_{\mathrm{R}}=0.1$ ), which is responsible for slow diffusion in frequency and space, we have set up a very difficult test case, which is more accentuated by the large thickness of the slab leading to multiple scattering effects. Since we have chosen a "local approximate Lambda operator", the non-locality of the problem causes more numbers of iterations, before these physical effects fully enter the source vector. Further, the core-wing separation scheme is not able to handle the diffusion in frequency space accurately in the wings (which is introduced by $R_{\mathrm{II}}$ redistribution), because scattering is assumed to be coherent in the wings. Thus the photon diffusion in the wings does not enter the solution through the source vector corrections, but instead only via the formal solver.

Thus, for most practical applications of line transfer, the core-wing separation scheme is very efficient, and works without any problem. The convergence of PALI on such a difficult 

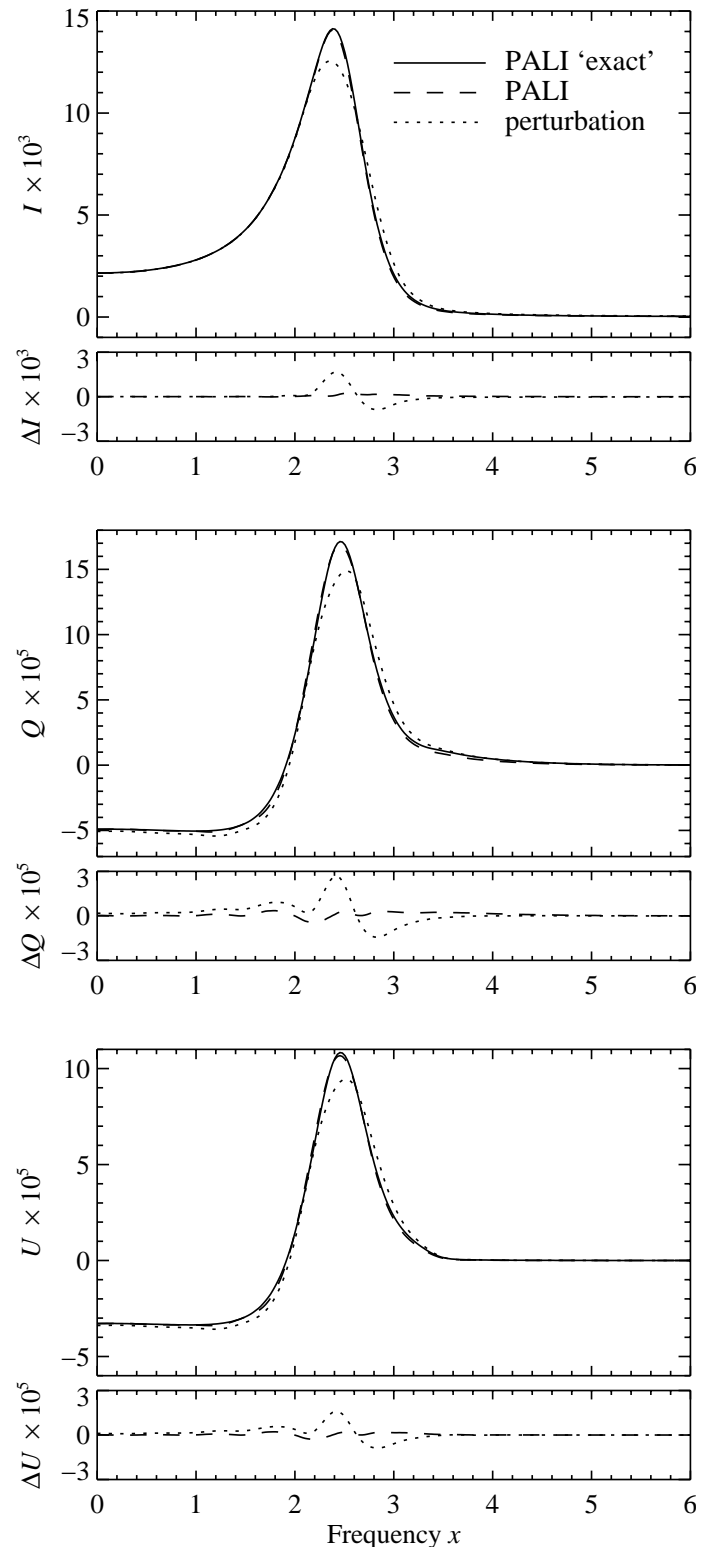

Fig. 4. Comparison of the emergent Stokes parameters for the direction $\mu=0.11$ and $\varphi=0^{\circ}$ computed using the PALI method developed in this paper and the perturbation method presented in Nagendra et al. (2002). An isothermal self-emitting slab is employed for these computations. The model parameters employed are $\left(T, a, \epsilon, \beta, \Gamma_{\mathrm{E}} / \Gamma_{\mathrm{R}}\right)=\left(2 \times 10^{2}, 10^{-3}, 10^{-4}, 0,10^{-1}\right)$ with the magnetic field parameters $\left(\Gamma_{B}, \theta_{B}, \varphi_{B}\right)=\left(1,30^{\circ}, 0^{\circ}\right)$. The line profiles obtained with PALI and the perturbation method on a coarse grid are compared to an "exact" PALI solution in which angle and frequency grids with a very fine resolution have been employed. Taking the moderate grid resolution into account the two methods agree well with each other.

problem as Hanle PRD with collisions, highlights the usefullness of this approach.

\subsection{Comparison with other numerical methods}

To validate the correctness of the method and study its accuracy we compare the new PALI method with an independent perturbation method (Nagendra et al. 2002).
The "perturbation method" handles the physical problem described in this paper by computing the scattering integral through an explicit use of angle-frequency quadratures. In the perturbation method, an unpolarized PRD solution is employed as starting solution, and the final result is obtained by treating Stokes $Q$ and $U$ parameters as perturbations to the intensity component $I$. However, because of the highly anisotropic nature of the redistribution matrices for the Hanle effect, a good resolution in angle and frequency space is necessary. This leads to large requirements of computer memory and CPU time to "accurately" compute the Stokes source vector by numerical quadrature.

\section{Model parameters}

The model parameters that we have employed are $\left(T, a, \epsilon, \beta, \Gamma_{\mathrm{E}} / \Gamma_{\mathrm{R}}\right)=\left(2 \times 10^{2}, 10^{-3}, 10^{-4}, 0,10^{-1}\right)$ with the magnetic field parameters $\left(\Gamma_{B}, \theta_{B}, \varphi_{B}\right)=\left(1,30^{\circ}, 0^{\circ}\right)$, using the physical domain structures shown in Fig. 1. We have used an isothermal self-emitting slab model with zero boundary conditions. Further we have chosen $W_{2}=1$ and $D^{(2)}=0.5 \Gamma_{\mathrm{E}}$.

For computing the case presented in Fig. 4 we choose a coarse angle and frequency grid, such that the computer requirements of the perturbation code remain reasonable. We have employed an optical depth resolution of 5 depth points per decade, 5 Gaussian latitude angles, 8 azimuth angles and a 31 point frequency grid with the last frequency point $x_{\max }=40$. Such a moderate resolution already required almost $1 \mathrm{~GB}$ of main memory and over 15 minutes of CPU time on a Alpha Server workstation for the perturbation code.

For comparison we run the PALI code also on a very fine grid with 11 Gaussian latitude angles, 115 frequency points, and also $x_{\max }=40$. We call the result obtained with this fine grid "exact", while the line profile computed by PALI with the moderate grid resolution is denoted by "coarse".

\section{Discussion}

A comparison between the emergent Stokes vector computed with the PALI method and the perturbation method (dotted) is shown in Fig. 4. Both the PALI results for the fine (solid) and for the coarse (dashed) grids are given. Below each panel of the Stokes parameters the absolute difference

$\Delta I=I($ exact $)-I($ coarse $)$

of the lines computed with the "exact" and the coarse grids is plotted (and analogous for Stokes $Q$ and $U$ ).

PALI yields very similar results for both grid resolutions. The emergent line profiles computed with the perturbation code differ up to 20 or $30 \%$ from the "exact" solution in the peak around $x=2.5$. This disagreement can be accounted for to the usage of the sparse grids. Because PALI makes use of an azimuthal Fourier expansion of the radiation field and the phase matrix it handles the azimuth dependence of the problem exactly. Note that the radiation field is in general not azimuthally symmetric when slanted, directed magnetic fields are present. On the other hand, the perturbation method involves explicit 
Table 1. CPU time and memory requirements for PALI and the perturbation methods. The model parameters are the same as specified in Sect. 4.2 and the caption for Fig. 4. The iteration sequence is stopped when the relative change of the Stokes $I$ source function and of the surface polarization fall below $10^{-3}$ in successive iteration steps. The computations have been performed on a Alpha Server DS20 $500 \mathrm{MHz}$ with a DECchip 21140 processor.

\begin{tabular}{lcc}
\hline \hline Method & CPU time [s] & memory [Mbyte] \\
\hline PALI & 4 & 8 \\
perturbation & 1050 & 880 \\
\hline
\end{tabular}

numerical evaluations of the scattering integral with a summation over the azimuth. For this reason it may differ slightly from the PALI solution.

However, the overall features are reproduced by both independent methods, indicating that the Hanle problem is properly implemented. The great advantage of PALI is that it is much faster and by far more economic with memory resources. Table 1 compares the CPU time and the memory requirements of the two methods for the specific model parameters of Fig. 4, when employing the same (coarse) grids.

The new PALI code is also tested on a variety of PRD problems which are simpler than the most general problem discussed here. It can easily reproduce the results for limiting cases such as Hanle CRD, non-magnetic resonance scattering polarization etc.

\subsection{Flux conservation test}

In this section we present a self-consistency check based on the energy conservation principle. We consider a conservative scattering atmosphere characterized by $\epsilon=0$ and $\beta=0$. In such a purely scattering atmosphere there is no true absorption and thermal emission. Thus the net flux remains constant at every depth point. In particular the sum of the incoming flux at the top surface $F_{\text {top,in }}$ and at the bottom surface $F_{\text {bot,in }}$ of a slab atmosphere must be equal to the sum of the outgoing fluxs $F_{\text {top,out }}$ and $F_{\text {bot,out }}$ respectively at the top and the bottom surfaces of the atmosphere. Flux conservation test states that the fractional error $f_{\mathrm{FC}-\text { test }}$ should be satisfied to a very high degree of accuracy, namely

$$
\begin{aligned}
f_{\mathrm{FC}-\mathrm{test}} & =\frac{\left(F_{\text {top,in }}+F_{\text {bot,in }}\right)-\left(F_{\text {top }, \text { out }}+F_{\text {bot,out }}\right)}{\left(F_{\text {top,in }}+F_{\text {bot,in }}\right)} \\
& \simeq 0 .
\end{aligned}
$$

In the above equation, we have defined the integrated flux on the half space as

$$
F=\int_{-\infty}^{+\infty} \mathrm{d} x \int_{0}^{2 \pi} \mathrm{d} \varphi \int_{0}^{+1} \mathrm{~d} \mu \mu I(x, \mu, \varphi) .
$$

Deviations of $f_{\mathrm{FC}-\mathrm{test}}$ from values like $10^{-10}$ for a typical test case, say, the one considered here, can be accounted for by two causes, namely by round-off errors resulting from arithmetic operations by the machine, and by poor normalization of the frequency redistribution functions, angular phase matrices, the profile function, and the quadrature weights etc.

Apart from performing the flux conservation test we would like to compare the PALI code with yet another independent method. We choose the Discrete Space Method (DSM). The details of this method can be found in Nagendra (1986, 1988, 1994, and references cited therein). The DSM is a finite difference discrete ordinate method, based on the first order form of the transfer equation, and produces highly accurate results. However, since the DSM is not yet extended to include weak magnetic fields, we restrict ourselves to the non-magnetic case.

In order to compare the results of PALI with DSM we had to simplify our treatment of frequency redistriubtion. The redistribution matrix employed for this test case has the form

$\hat{\mathscr{R}}\left(x, x^{\prime}, \boldsymbol{n}, \boldsymbol{n}^{\prime}, \boldsymbol{B}\right)=(1-\epsilon) R\left(x, x^{\prime}\right) \hat{\boldsymbol{P}}_{\mathrm{R}}\left(\boldsymbol{n}, \boldsymbol{n}^{\prime}, W_{2}\right)$,

where $R$ can be chosen as either $R_{\mathrm{II}}, R_{\mathrm{III}}$, or as $\phi(x) \phi\left(x^{\prime}\right)$, representing CRD.

\section{Model parameters}

We use an isothermal, non-magnetic conservative scattering slab medium characterized by the parameters $(T, a, \epsilon, \beta)=$ $\left(2 \times 10^{4}, 10^{-3}, 0,0\right)$. At the upper boundary of the slab no radiation is incident while at the lower boundary an unpolarized radiation field is incident that illuminates the slab isotropically as defined in Eq. (69). We have set $W_{2}=1$. Depolarizing collisions have been neglected. We have used an optical depth grid with 4 depth points per decade, 5 Gaussian latitude angles, and a 31 point frequency grid with the last frequency point $x_{\max }=10.5$.

Again the results obtained on this rather coarse grid are compared to the PALI solution using very fine grids, namely 11 Gaussian latitude angles, 103 frequency points, but also with $x_{\max }=10.5$ for comparison reasons. The results based on the fine resolution are called "exact".

\section{Discussion}

The emergent Stokes profiles shown on the left hand side panel (a) of Fig. 5 refer to the angle averaged frequency redistribution function $R_{\mathrm{III}}$, while the right hand side panel (b) refers to the case of pure $R_{\mathrm{II}}$. The Stokes $U$ parameter is identically zero due to the absence of magnetic fields. We compare the results of the PALI method (dashed line) with those computed from the DSM (dots) using the coarse angle and frequency grids. These curves are compared to the "exact" solution from PALI in which the fine grids have been employed.

The Stokes vector obtained by the two methods on the coarse grids coincide very well except for slight differences in the sharp peak of Stokes $Q$ in the $R_{\mathrm{III}}$ case. Since the differences are of the same order as when comparing the results from coarse and exact grids, they can be accounted for by the usage of the moderate grid resolution. We emphasize that the coarse grid was chosen because the DSM method requires large amount of computer memory, as the redistribution matrix, 
(a) $R_{\mathrm{III}}$ frequency redistribution
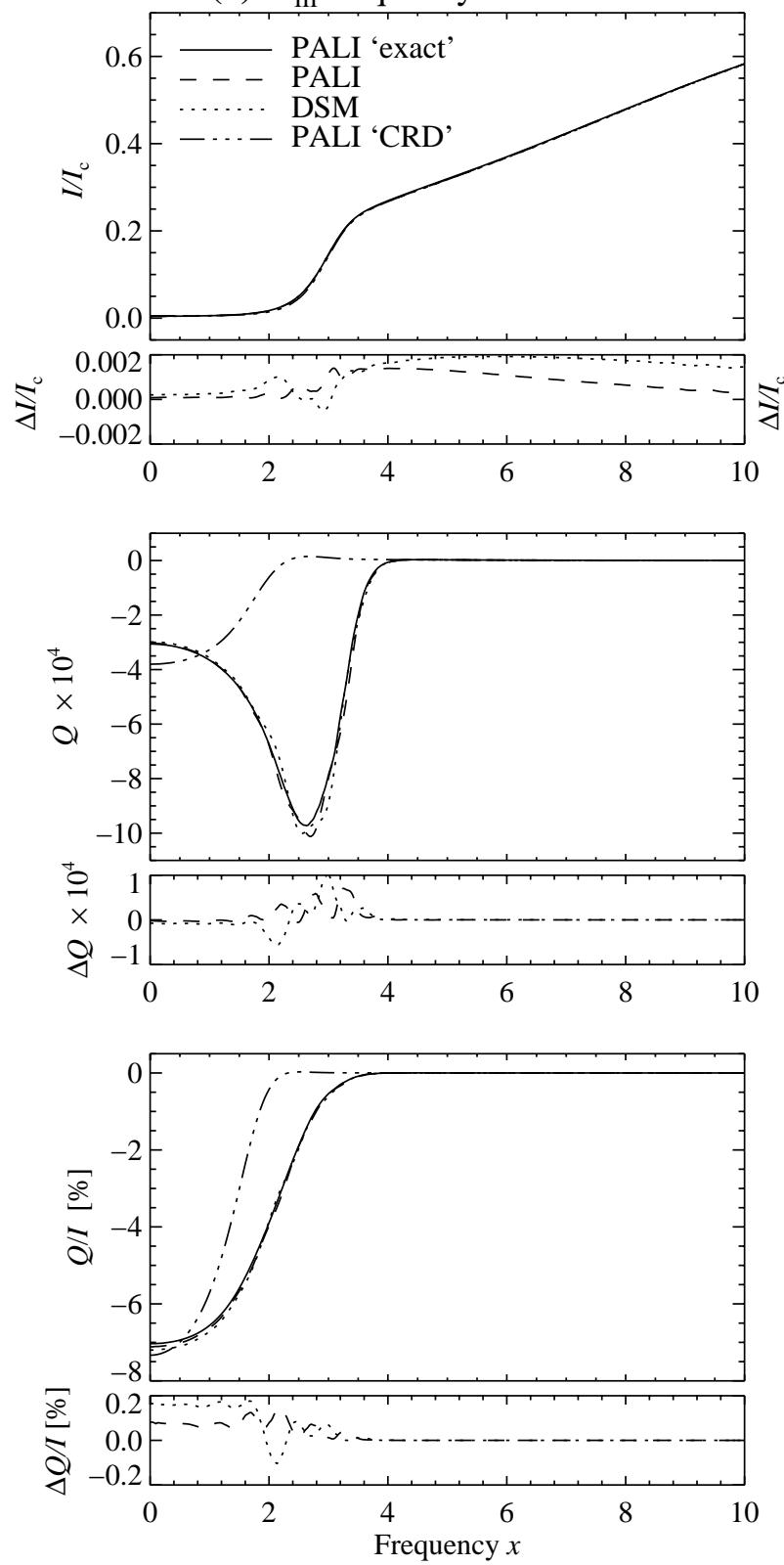

(b) $R_{\mathrm{II}}$ frequency redistribution
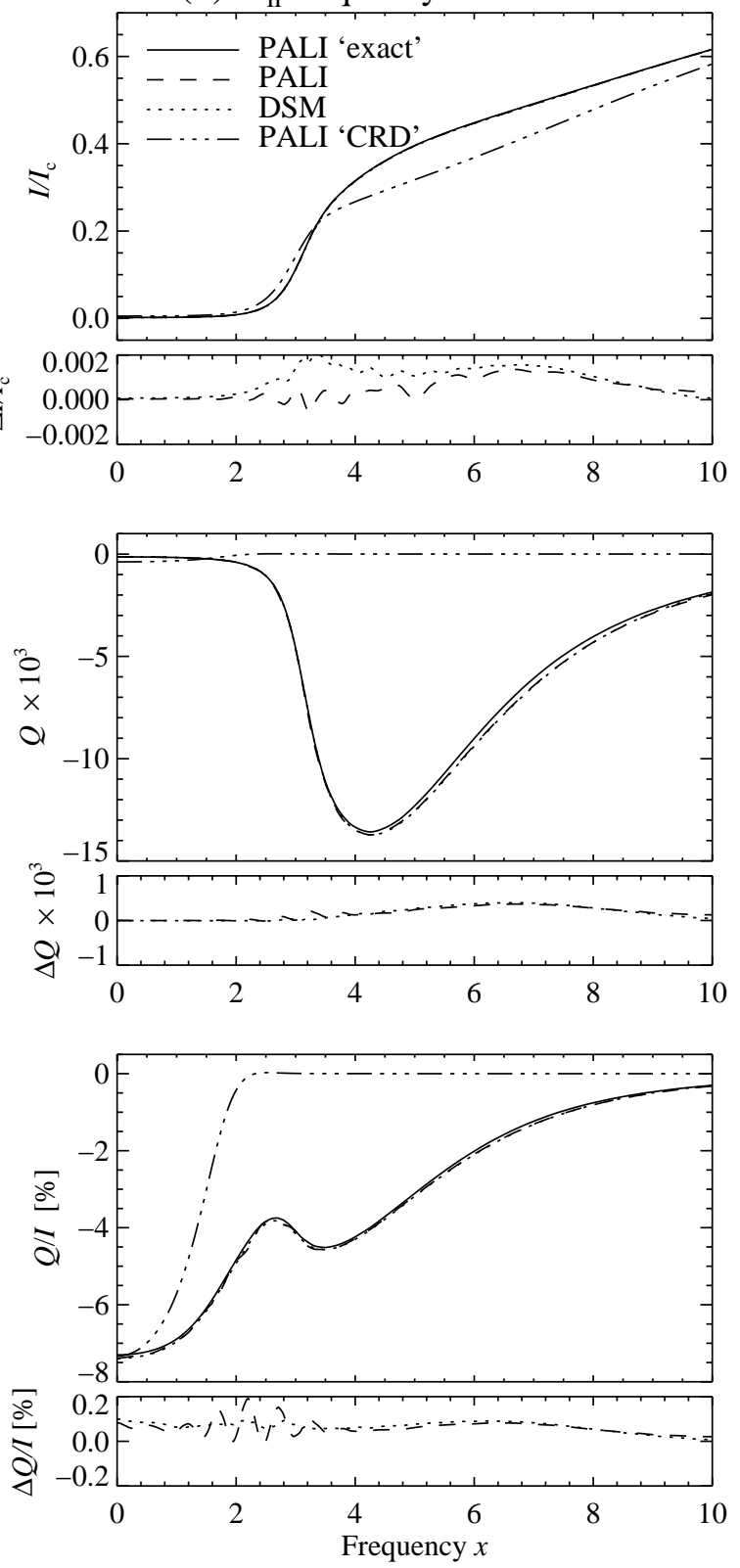

Fig. 5. Comparison of the DSM (dots) and PALI (dashed) methods of solution for a conservative scattering, non-magnetic atmosphere, illuminated from below. The relative difference of these line profiles from the PALI result obtained on a very fine grid (solid) is plotted below the panels of the Stokes parameters. Emergent Stokes parameters are shown for the direction $\mu=0.047$ and $\varphi=0^{\circ}$. The model parameters are $(T, a, \epsilon, \beta)=\left(2 \times 10^{4}, 10^{-3}, 0,0\right)$. Due to axial symmetry, Stokes $U \equiv 0$. Panel a) on the left side refers to the profiles computed using the frequency redistribution function $R_{\mathrm{III}}$, while the right side panel $\mathbf{b}$ ) refers to $R_{\mathrm{II}}$. For comparison we give the CRD result of PALI (dash-tripledotted).

phase matrix, and several auxiliary matrices in the numerical algorithm have to be stored in the main memory. Further, the CPU time for the DSM scales in the same manner as the Feautrier method.

Obviously, the coarse frequency grid is not good enough in this semi-infinite case, if we wish to calculate observed line profiles. But the main purpose of Fig. 5 was to demonstrate that two entirely independent methods of solution give reasonably matching results in the difficult case of a pure scattering atmosphere. At least it proves the correctness of our handling of the PRD function in these methods.
The cause of the sharp feature of Stokes $Q$ in the $R_{\mathrm{III}}$ case is to be found in the nature of the $R_{\mathrm{III}}$ function itself. Frequently $R_{\mathrm{III}}$ is approximated by CRD. For frequencies $x>5$ the $R_{\mathrm{III}}$ function indeed greatly resembles CRD. But in the line core, and especially in the near wings $2<x<4$, it differs substantially from CRD (see e.g. Mihalas 1978, p. 432). In this range of frequencies, the frequency coherence contributes to the appearance of a weak polarization feature in the $Q$ profile. Note that Stokes $Q$ is much smaller compared to the $R_{\mathrm{II}}$ case. In the $Q / I$ profile the peak only leads to a widening of the core as compared to CRD, not to a separate maximum. The CRD result 
from PALI (dash-triple-dotted), employing the fine grids, is given in all panels of Fig. 5. The intensity profiles for CRD and $R_{\mathrm{III}}$ coincide.

Due to the well-known wing coherences of the $R_{\mathrm{II}}$ function, which manifest themselve for all frequencies $x>3$, the degree of polarization is larger in the $R_{\mathrm{II}}$ than in the $R_{\mathrm{III}}$ case. However, the near wing maxima in the $R_{\text {II }}$ case are not sharp because of the coherence at all wing frequencies, in contrast to $R_{\mathrm{III}}$ where the coherence behavior is confined to the intermediate frequencies $2<x<4$. The smooth superposition of wing coherence peaks of $R_{\mathrm{II}}$ ensure the broad, smooth maximum in the $Q$ profile that is even present in $Q / I$. The Stokes $Q$ parameter slowly approaches zero only in the far wings $x>10$.

The flux conservation test was passed by both the PALI and the DSM method. The quantity $f_{\mathrm{FC}-\text { test }}$ was of the order of $10^{-11}$ for PALI and $10^{-8}$ for DSM. The accuracy of the DSM appears somewhat lesser because of accumulated round-off errors, which eat into the solution. However, it is easy to remedy this by working in the DSM with finer grid resolution and a stronger step size criteria, which in turn increase the CPU time requirement (!). These are well known practical problems of working with finite difference methods. The flux conservation test clearly demonstrates that no spurious sources and sinks are added in the process of iterations, and that the PALI method gives results to the machine accuracy.

\section{Conclusions}

In this paper we have proposed a new Polarized Approximate Lambda Iteration (PALI) method, which is derived through a generalization of the PALI method presented in Nagendra et al. (1999). This basically involved a generalization of the corewing separation approach to incorporate the logical frequency domains in $\left(x, x^{\prime}\right)$-space, recently derived by Bommier (1997b) in her formulation of the Hanle redistribution matrix including PRD and collisions. It is shown that this exact formulation can now be incorporated into the existing polarized PRD line transfer codes which can solve the problem of Hanle scattering. The proposed method is well tested for its numerical performance, and the tests may serve as benchmarks for future work in this area.

Acknowledgements. We would like to thank I. Hubeny for comments that helped to improve the manuscript. D. M. Fluri would like to thank J. O. Stenflo for his support and for making it possible to visit the Indian Institute of Astrophysics (IIA) in Bangalore, India. Further he would like to thank the Director and all members of IIA for the kind support and hospitality during two extended visits. Special thanks go to Peter Messmer for his help running the perturbation code on a NEC SX-5 machine. Support for the work of D. M. Fluri has been obtained through grant no. 20-64945.01 from the Swiss National Science Foundation, which is gratefully acknowledged. K. N. Nagendra would like to thank H. Frisch, of the Observatoire de la Côte d'Azur, for organizing visits to Nice, during which part of this work was completed.

\section{References}

Bommier, V. 1997a, A\&A, 328, 706

Bommier, V. 1997b, A\&A, 328, 726

Bommier, V., \& Sahal-Bréchot, S. 1978, A\&A, 69, 57

Bommier, V., \& Stenflo, J. O. 1999, A\&A, 350, 327

Dittman, O. J. 1999, in Solar Polarization, Proc. 2nd SPW, ed. K. N. Nagendra, \& J. O. Stenflo (Dordrecht: Kluwer), ASSL, 243, 201

Domke, H., \& Hubeny, I. 1988, ApJ, 334, 527

Fabiani Bendicho, P., \& Trujillo Bueno, J. 1999, in Solar Polarization, Proc. 2nd SPW, ed. K. N. Nagendra, \& J. O. Stenflo (Dordrecht: Kluwer), ASSL, 243, 219

Faurobert, M. 1987, A\&A, 178, 269

Faurobert-Scholl, M. 1991, A\&A, 246, 469

Faurobert-Scholl, M. 1992, A\&A, 258, 521

Faurobert-Scholl, M. 1993, A\&A, 268, 765

Faurobert-Scholl, M. 1996, in Solar Polarization, Proc. 1st SPW, ed. J. O. Stenflo, \& K. N. Nagendra (Dordrecht: Kluwer), Sol. Phys., 164,79

Faurobert-Scholl, M., Feautrier, N., Machefert, F., Petrovay, K., \& Spielfiedel, A. 1995, A\&A, 298, 289

Faurobert-Scholl, M., Frisch, H., \& Nagendra, K. N. 1997, A\&A, 322, 896, Paper I

Faurobert-Scholl, M., Paletou, F., \& Bommier, V. 1999, in Solar Polarization, Proc. 2nd SPW, ed. K. N. Nagendra \& J. O. Stenflo (Dordrecht: Kluwer), ASSL, 243, 115

Frisch, H. 1998, A\&A, 338, 683

Frisch, H. 1999, in Solar Polarization, Proc. 2nd SPW, ed. K. N. Nagendra, \& J. O. Stenflo (Dordrecht: Kluwer), ASSL, 243, 97

Gandorfer, A. 2000, The Second Solar Spectrum, Vol I: $4625 \AA$ to $6995 \AA$, ISBN no. 3728127647 (Zurich: VdF)

Heinzel, P. 1981, J. Quant. Spectrosc. Radiat. Transfer, 25, 483

Hubeny, I. 1982, J. Quant. Spectrosc. Radiat. Transfer, 27, 593

Hubeny, I. 1985, in Progress in Stellar Spectral Line Fromation theory, ed. J. E. Beckman, \& L. Crivellari (Dordrecht: Reidel), 27

Hubeny, I., \& Cooper, J. 1986, ApJ, 305, 852

Hubeny, I., \& Lites, B. W. 1995, ApJ, 455, 376

Hubeny, I., Oxenius, J., \& Simonneau, E. 1983a, J. Quant. Spectrosc. Radiat. Transfer, 29, 477

Hubeny, I., Oxenius, J., \& Simonneau, E. 1983b, J. Quant. Spectrosc. Radiat. Transfer, 29, 495

Hummer, D. G. 1962, MNRAS, 125, 21

Ignace, R. 2001, ApJ, 547, 393

Ignace, R., Cassinelli, J. P., \& Nordsieck, K. H. 1999, ApJ, 520, 335

Ignace, R., Nordsieck, K. H., \& Cassinelli, J. P. 1997, ApJ, 486, 550

Landi Degl'Innocenti, E. 1983, Sol. Phys., 85, 3

Landi Degl'Innocenti, E. 1984, Sol. Phys., 91, 1

Landi Degl'Innocenti, E. 1985, Sol. Phys., 102, 1

Landi Degl'Innocenti, E. 1998, Nature, 392, 256

Landi Degl'Innocenti, E., Landi Degl'Innocenti, M., \& Landolfi, M. 1997, in Froum THEMIS, Science with THEMIS, ed. N. Mein, \& S. Sahal Bréchot, Paris Obs. Publ., 59

Landi Degl'Innocenti, M., \& Landi Degl'Innocenti, E. 1988, A\&A, 192,374

Manso Sainz, R., \& Trujillo Bueno, J. 1999, in Solar Polarization, Proc. 2nd SPW, ed. K. N. Nagendra, \& J. O. Stenflo (Dordrecht: Kluwer), ASSL, 243, 143

Manso Sainz, R., \& Trujillo Bueno, J. 2001, in Advanced Solar Polarimetry - Theory, Observations, and Instrumentation, ed. M. Sigwarth, ASP Conf. Ser., 236, 213

Mihalas, D. 1978, Stellar Atmospheres, 2nd edn. (San Francisco: Freeman and Company)

Nagendra, K. N. 1986, PhD thesis, Bangalore University, Bangalore, India 
Nagendra, K. N. 1988, ApJ, 335, 269

Nagendra, K. N. 1994, ApJ, 432, 274

Nagendra, K. N. 1995, MNRAS, 274, 523

Nagendra, K. N., Frisch, H., \& Faurobert, M. 2002, A\&A, 395, 305

Nagendra, K. N., Frisch, H., \& Faurobert-Scholl, M. 1998, A\&A, 332, 610, Paper III

Nagendra, K. N., Frisch, H., Faurobert-Scholl, M., \& Paletou, F. 2000, J. Astrophys. Astron., 21, 255, Paper V

Nagendra, K. N., Paletou, F., Frisch, H., \& Faurobert-Scholl, M. 1999, in Solar Polarization, Proc. 2nd SPW, ed. K. N. Nagendra, \& J. O. Stenflo (Dordrecht: Kluwer), ASSL, 243, 127, Paper IV

Nagendra, K. N., \& Stenflo, J. O., eds. 1999, Solar Polarization, Proc. 2nd SPW (Dordrecht: Kluwer), ASSL, 243

Olson, G. L., Auer, L. H., \& Buchler, J. R. 1986, J. Quant. Spectrosc. Radiat. Transfer, 35, 431

Omont, A., Smith, E. W., \& Cooper, J. 1972, ApJ, 175, 185

Omont, A., Smith, E. W., \& Cooper, J. 1973, ApJ, 182, 283

Oxenius, J. 1965, J. Quant. Spectrosc. Radiat. Transfer, 5, 771

Paletou, F., \& Auer, L. H. 1995, A\&A, 297, 771

Paletou, F., \& Faurobert-Scholl, M. 1997, A\&A, 328, 343, Paper II
Rees, D. E., \& Saliba, G. J. 1982, A\&A, 115, 1

Stenflo, J. O. 2001, in Advanced Solar Polarimetry - Theory, Observations, and Instrumentation, ed. M. Sigwarth, ASP Conf. Ser., 236, 97

Stenflo, J. O., Gandorfer, A., Wenzler, T., \& Keller, C. U. 2001, A\&A, 367,1033

Stenflo, J. O., \& Keller, C. U. 1996, Nature, 382, 588

Stenflo, J. O., \& Keller, C. U. 1997, A\&A, 321, 927

Stenflo, J. O., Keller, C. U., \& Gandorfer, A. 2000, A\&A, 355, 789

Stenflo, J. O., \& Nagendra, K. N., eds. 1996, Solar Polarization, Proc. 1st SPW (Dordrecht: Kluwer), Sol. Phys., 164

Stenflo, J. O., Twerenbold, D., \& Harvey, J. W. 1983a, A\&AS, 52, 161

Stenflo, J. O., Twerenbold, D., Harvey, J. W., \& Brault, J. W. 1983b, A\&AS, 54, 505

Streater, A., Cooper, J., \& Rees, D. E. 1988, ApJ, 335, 503

Trujillo Bueno, J. 2001, in Advanced Solar Polarimetry - Theory, Observations, and Instrumentation, ed. M. Sigwarth, ASP Conf. Ser., 236, 161

Trujillo Bueno, J., \& Landi Degl'Innocenti, E. 1997, ApJ, 482, L183

Trujillo Bueno, J., \& Manso Sainz, R. 1999, ApJ, 516, 436 